Article

\title{
Genome-Wide Analysis and Expression Profiling of Rice Hybrid Proline-Rich Proteins in Response to Biotic and Abiotic Stresses, and Hormone Treatment
}

\author{
Ritu Kapoor ${ }^{1}$, Gulshan Kumar ${ }^{2}$, Preeti Arya ${ }^{2} \odot$, Rajdeep Jaswal ${ }^{2,3}$, Priyanka Jain ${ }^{4}$, \\ Kashmir Singh 1,*(D) and Tilak Raj Sharma ${ }^{2, *}$ \\ 1 Department of Biotechnology, Panjab University, Chandigarh 160014, Punjab, India; \\ ritukapoor1985@gmail.com \\ 2 National Agri-Food Biotechnology Institute, Mohali 140306, Punjab, India; gulshan.ihbt@gmail.com (G.K.); \\ arya.preet9@gmail.com (P.A.); rajdeepjaswal52@gmail.com (R.J.) \\ 3 Department of Microbiology, Panjab University, Chandigarh 160014, Punjab, India \\ 4 National Institute of Plant Biotechnology, New Delhi 110012, India; priybioinfo@gmail.com \\ * Correspondence: kashmirbio@pu.ac.in (K.S.); trsharma@nabi.res.in (T.R.S.); Tel.: +91-172-5221300 (T.R.S.)
}

Received: 29 June 2019; Accepted: 22 August 2019; Published: 11 September 2019

\begin{abstract}
Hybrid proline-rich proteins (HyPRPs) belong to the family of 8-cysteine motif (8CM) containing proteins that play important roles in plant development processes, and tolerance to biotic and abiotic stresses. To gain insight into the rice HyPRPs, we performed a systematic genome-wide analysis and identified 45 OsHyPRP genes encoding 46 OsHyPRP proteins. The phylogenetic relationships of OsHyPRP proteins with monocots (maize, sorghum, and Brachypodium) and a dicot (Arabidopsis) showed clustering of the majority of OsHyPRPs along with those from other monocots, which suggests lineage-specific evolution of monocots HyPRPs. Based on our previous RNA-Seq study, we selected differentially expressed OsHyPRPS genes and used quantitative real-time-PCR (qRT-PCR) to measure their transcriptional responses to biotic (Magnaporthe oryzae) and abiotic (heat, cold, and salt) stresses and hormone treatment (Abscisic acid; ABA, Methyl-Jasmonate; MeJA, and Salicylic acid; SA) in rice blast susceptible Pusa Basmati-1 (PB1) and blast-resistant near-isogenic line PB1+Pi9. The induction of OsHyPRP16 expression in response to the majority of stresses and hormonal treatments was highly correlated with the number of cis-regulatory elements present in its promoter region. In silico docking analysis of OsHyPRP16 showed its interaction with sterols of fungal/protozoan origin. The characterization of the OsHyPRP gene family enables us to recognize the plausible role of OsHyPRP16 in stress tolerance.
\end{abstract}

Keywords: rice; hybrid proline-rich proteins; biotic stress; abiotic stress; Magnaporthe oryzae

\section{Introduction}

Being sessile in nature, plants are continuously exposed to biotic and abiotic stresses that adversely affect their productivity. The plant defense response against biotic stresses can be categorized into pathogen-associated molecular patterns (PAMP)-triggered immunity (PTI) and effector-triggered immunity (ETI) [1]. In both PTI and ETI, the activation of a complex network of signaling cascade pathways leads to the induction of resistance response mediated through pathogenesis-related $(P R)$ genes, reactive oxygen species (ROS) and secondary metabolites [2]. The initiation of PR proteins against various biotic stresses has been reported in different plant species [3,4]. Among the 17 classes of PR proteins [5], the 8-cysteine motif (8CM) containing PR proteins belong to the prolamin superfamily, which can be further categorized into subfamilies including non-specific lipid transfer proteins (nsLTPs), $2 \mathrm{~S}$ albumins, alpha-amylase/trypsin inhibitors, and hydrophobic seed proteins [6,7]. The 8CM is 
usually 90-100 amino acid residues long with a conserved pattern of cysteine residues spaced as $C-X_{n}-C-X_{n}-C C-X_{n}-C X C-X_{n}-C-X_{n}-C[6,8]$. The $8 C M$ proteins are different from the other eight cysteine residue-containing proteins, such as lectins and agglutinin, based on the size, the pattern of cysteine spacing, an array of disulfide bonds, and hydrophobicity profile [9].

In several $8 \mathrm{CM}$ containing proteins, the proline-rich repeats precede the $8 \mathrm{CM}$, and such proteins are classified as HyPRPs. However, the N-terminal domain of HyPRPs resembles the structural cell-wall proline-rich proteins (PRPs) $[10,11]$, they lack the characteristic amino acid motifs of PRPs and are highly variable in their size and proline repeats composition [8,12]. The presence of atypical $\mathrm{N}$-terminal proline-rich repeat domain and typical C-terminal 8CM domain categorize the HyPRPs together with the $8 \mathrm{CM}$ containing proteins of prolamin superfamily [8]. The structural analysis of a typical $8 \mathrm{CM}$ domain-containing protein, nsLTP, revealed that four disulfide bonds between eight cysteine residues are essential for a stable hydrophobic cavity that can accommodate various lipids and hydrophobic ligands with high specificity and plasticity [13-15].

The expression profile of $H y P R P s$ has been primarily used to infer their functions. For example, the stress-inducible expression of BnPRP (Brassica napus), CcHyPRP (Cajanus cajan), EARLI1 (Arabidopsis), GhHyPRP3 (Gossypium hirsutum), GhHyPRP4, JsPRP1 (Juglans sigillata), MfHyPRP (Medicago falcata), MsPRP2 (Medicago sativa) suggest their role in abiotic stress tolerance. [16-24]. Similarly, the induced expression of AtDHyPRP1 (Arabidopsis), CcHyPRP, JsPRP1, GmHyPRP (Glycine max) and SbPRP (Glycine max) in response to bacterial or fungal or viral pathogens indicate their role towards biotic stresses [24-28]. In response to various signaling molecules such as phytohormones, $\mathrm{H}_{2} \mathrm{O}_{2}, \mathrm{NO}$, and other oxidative stress-inducing molecules, the altered expression of HyPRPs including JSPRP1, $M F H y P R P, S b P R P$, and SlHyPRP1 (Solanum lycopersicum), suggests their role either directly or indirectly in the defense-related signaling pathways [23,24,26,29].

The functional analysis of HyPRPS suggests their diverse roles in biotic and abiotic stress tolerance, morphogenesis, and cellular and developmental processes $[9,19,20,30-34]$. The functional characterization of HyPRP genes, including EARLI1, PtrPRP (Poncirus trifoliata), and GhHyPRP4, suggests their positive role in the freezing or cold tolerance $[19,21,35,36]$. The overexpression of CCHyPRP, GhHyPRP3, JsPRP1, and MFHyPRP were found to enhance tolerance toward multiple abiotic stresses, such as salinity and cold stress [20,22,23,37]. In contrast, tomato SlHyPRP1 and SpHyPRP1 (Solanum pennellii) were found to negatively regulate the stress response against drought, salt, and oxidative stresses in tomato [29]. The Arabidopsis HyPRP, AZI1, was found to play an essential role as a vital component in systemic acquired resistance (SAR), during Pseudomonas syringae infection [38]. Similarly, tobacco overexpressing JSPRP1 exhibited strong resistance against Colletotrichum gloeosporioides infection [24]. Conversely, the CaHyPRP1 (Capsicum annuum) and GbHyPRP1 (Gossypium barbadense) were found to negatively regulate the basal defense during biotic stresses, possibly through suppression of $\operatorname{ROS}[39,40]$. All the above studies highlighted the importance of HyPRPs in biotic and abiotic responses, and therefore, it is of great significance to identify and characterize the HyPRPs in plants. Although Dvořáková et al. [12] identified members of the HyPRPs gene family in seven plant species, including rice, they explained the comparative sequence diversity among the HyPRPs gene family members.

Rice (Oryza sativa L.) is a cereal that is widely consumed as a staple food; therefore, sustainable rice production is a key factor towards insuring food security for our world's continuously-growing population. It is essential to reduce the losses associated with various biotic and abiotic stresses to achieve sustainability in rice production. Owing to the significance of HyPRPs in biotic and abiotic stress tolerance, in the present investigation, we carried out genome-wide identification, comparative phylogeny and duplication analysis on the HyPRP gene family in rice. Furthermore, we performed the qRT-PCR based expression profiling of rice HyPRPs genes in response to biotic and abiotic stresses, and phytohormone treatment in rice blast susceptible PB1 and near-isogenic blast-resistant rice line PB1+Pi9 [41-43]. The present investigation provides a basic framework that can be explored to unravel 
the biological significance of rice HyPRPs and to target the putative candidate genes of this $8 \mathrm{CM}$ gene family to devise strategies for the development of climate-resilient crop plants.

\section{Results and Discussion}

\subsection{Identification and Annotation of Putative OsHyPRPs in Rice}

The scanning of hidden Markov model (HMM) profile resulted in the identification of 65, 156 and 134 proteins containing Hydrophob_seed (PF14547), Tryp_alpha_amyl (PF00234), and LTP_2 (PF14368) domains, respectively. The PfamScan was used to confirm the presence of these domains, which resulted in the identification of 50, 130, and 131 proteins with Hydrophob_seed domain, Tryp_alpha_amyl domain, and LTP_2 domain, respectively. By combining the results, a total of 179 proteins were detected as unique proteins, where four proteins (LOC_Os07g11310.1, LOC_Os07g12080.1, LOC_Os07g11630.1, and LOC_Os07g11650.1) based on the BLAST similarity were found to be trypsin-alpha amylase inhibitors or cereal storage proteins and therefore removed from the further analysis. We manually inspected the sequences of the remaining 175 candidate proteins and excluded 126 proteins, which lacked proline-rich repeats and $8 \mathrm{CM}$. Additionally, we removed three proteins without $\mathrm{N}$-terminal signal peptides. Using this stringent process, we identified a total of 46 putative OsHyPRPs encoded by $45 \mathrm{OsHyPRPs}$ genes in rice, which showed characteristic $\mathrm{N}$-terminal signal peptides followed by proline residues repeats and C-terminal conserved $8 \mathrm{CM}$ motifs. However, in the previous studies by Dvorakova et al. [12] and Boutrot et al. [44], identified 31 and 21 genes, respectively, encoding HyPRPs in the whole rice genome. Such a difference in the number of identified HyPRPS may be due to the use of different identification approaches and the continuous refinement of the rice genome. The multiple sequence alignment of OsHyPRP protein sequences showed the conservation of the eight-cysteine skeleton in the $8 \mathrm{CM}$ region, while the proline-rich region was found to be highly non-conserved (Figure 1).

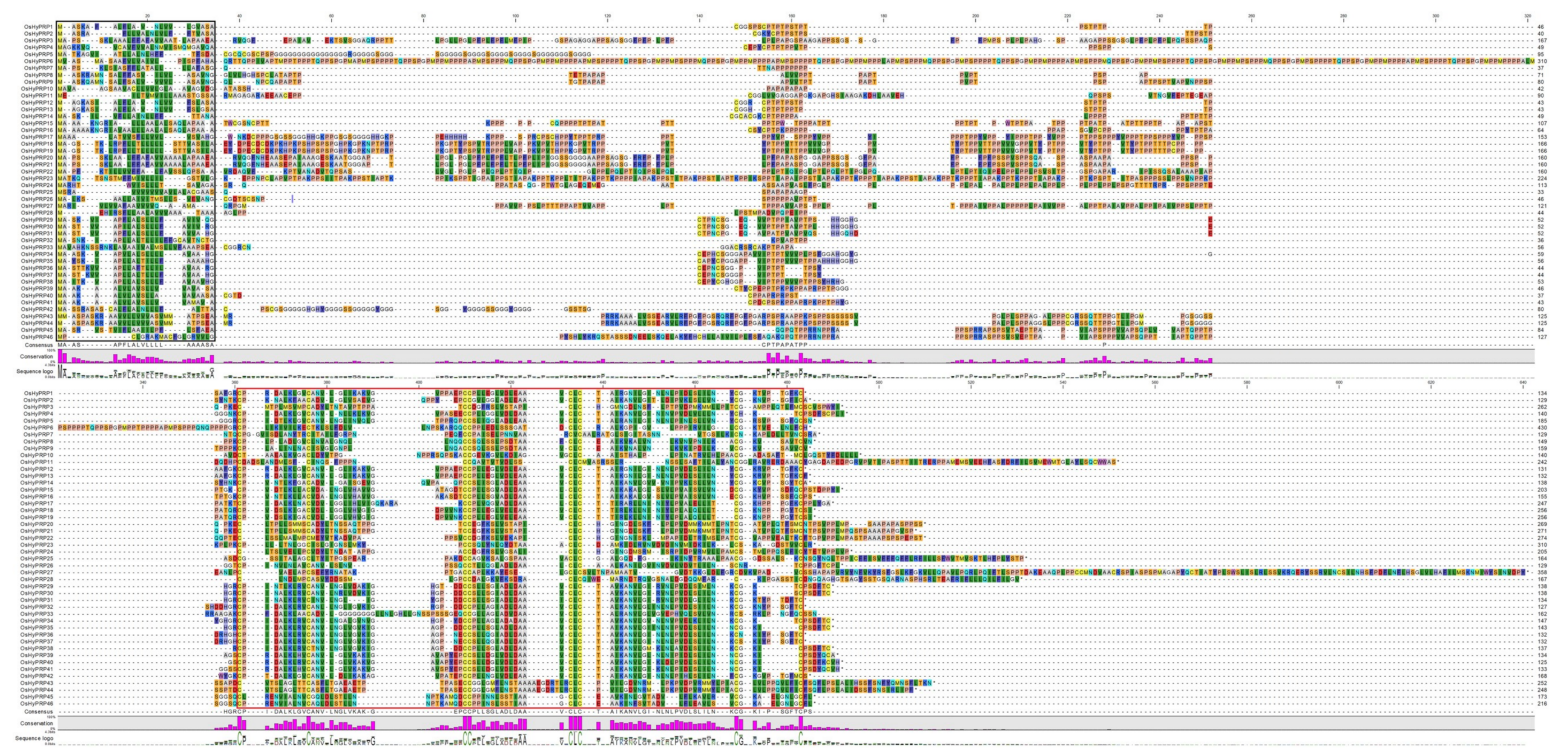

Figure 1. The multiple sequence alignment of OsHyPRPs showing conserved regions. The conserved amino acid residues in the black box are N-terminal signal peptides (may extend further for several proteins due to alignment constrains), while the C-terminal 8CM of OsHyPRPs is marked in the red box. The $8 \mathrm{CM}$ region is typical to the other $8 \mathrm{CM}$ protein with a conserved eight cysteine skeleton. The region in-between the black and red boxes showed the non-conserved proline-rich region, which is atypical to the proline-rich cell wall structural proteins. 
The Physico-chemical characteristics of the 46 identified OsHyPRPs are summarized in Table 1. The average molecular mass and the theoretical $\mathrm{pI}$ of mature OsHyPRP proteins was estimated to be $16.3 \mathrm{kDa}$ and $7.3 \mathrm{pI}$, respectively. The signal peptide length for OsHyPRP proteins varied from 20 to 32 amino acid residues. Using signalP, we predict that the majority of OsHyPRPs targets to the secretory pathway, with few exceptions, where OsHyPRP24 and OsHyPRP46 target to the mitochondria, while OsHyPRP43, OsHyPRP44, and OsHyPRP45 target to the chloroplast (Table 1).

Table 1. List of Hybrid proline-rich proteins (HyPRPs) in rice and some of their features.

\begin{tabular}{|c|c|c|c|c|c|c|}
\hline \multirow{2}{*}{ Locus IDs } & \multirow{2}{*}{ Nomenclature } & \multicolumn{2}{|c|}{ Signal Peptide } & \multicolumn{3}{|c|}{ Mature Protein } \\
\hline & & Amino Acid & Target $^{a}$ & Amino Acid & Mass (kDa) & pI \\
\hline LOC_Os02g44310.1 & OsHyPRP1 & 22 & $S$ & 111 & 11.27 & 8.14 \\
\hline LOC_Os02g44320.1 & OsHyPRP2 & 22 & $S$ & 104 & 10.65 & 6.24 \\
\hline LOC_Os02g49280.1 & OsHyPRP3 & 28 & $S$ & 233 & 23.6 & 5.43 \\
\hline LOC_Os03g01300.1 & OsHyPRP4 & 29 & $S$ & 110 & 11.32 & 5.04 \\
\hline LOC_Os03g01320.1 & OsHyPRP5 & 24 & $S$ & 160 & 13.97 & 8.37 \\
\hline LOC_Os03g14615.1 & OsHyPRP6 & 25 & $S$ & 404 & 41.07 & 8.91 \\
\hline LOC_Os03g14630.1 & OsHyPRP7 & 25 & $S$ & 103 & 10.66 & 8.50 \\
\hline LOC_Os03g14642.1 & OsHyPRP8 & 26 & $S$ & 122 & 12.29 & 8.15 \\
\hline LOC_Os03g14654.1 & OsHyPRP9 & 26 & $S$ & 132 & 13.1 & 8.44 \\
\hline LOC_Os03g26800.1 & OsHyPRP10 & 27 & $S$ & 112 & 11.16 & 6.20 \\
\hline LOC_Os03g43050.1 & OsHyPRP11 & 20 & S & 221 & 23 & 4.69 \\
\hline LOC_Os04g46810.1 & OsHyPRP12 & 23 & $S$ & 105 & 10.8 & 8.65 \\
\hline LOC_Os04g46820.1 & OsHyPRP13 & 23 & $S$ & 106 & 10.91 & 8.43 \\
\hline LOC_Os04g46830.1 & OsHyPRP14 & 22 & $S$ & 115 & 7.4 & 8.02 \\
\hline LOC_Os04g52250.1 & OsHyPRP15 & 27 & $S$ & 175 & 17.56 & 4.88 \\
\hline LOC_Os04g52260.1 & OsHyPRP16 & 32 & $S$ & 122 & 12.18 & 8.04 \\
\hline LOC_Os06g07220.1 & OsHyPRP17 & 22 & $\mathrm{~S}$ & 224 & 23.35 & 8.96 \\
\hline LOC_Os06g43600.1 & OsHyPRP18 & 27 & S & 228 & 23.89 & 8.86 \\
\hline LOC_Os06g43600.2 & OsHyPRP19 & 27 & $S$ & 228 & 23.89 & 8.86 \\
\hline LOC_Os06g46780.1 & OsHyPRP20 & 28 & S & 240 & 23.79 & 5.51 \\
\hline LOC_Os06g46870.1 & OsHyPRP21 & 28 & $S$ & 242 & 23.98 & 5.51 \\
\hline LOC_Os07g29230.1 & OsHyPRP22 & 26 & $S$ & 247 & 25.26 & 5.24 \\
\hline LOC_Os07g37385.1 & OsHyPRP23 & 26 & $S$ & 283 & 28.86 & 9.89 \\
\hline LOC_Os07g39640.1 & OsHyPRP24 & 19 & M & 186 & 19.14 & 4.72 \\
\hline LOC_Os07g43290.2 & OsHyPRP25 & 22 & $\mathrm{~S}$ & 141 & 14.61 & 8.40 \\
\hline LOC_Os10g09920.1 & OsHyPRP26 & 25 & $S$ & 103 & 10.36 & 3.97 \\
\hline LOC_Os10g11370.1 & OsHyPRP27 & 21 & $S$ & 336 & 35.39 & 8.88 \\
\hline LOC_Os10g11730.1 & OsHyPRP28 & 23 & $S$ & 143 & 15.18 & 5.15 \\
\hline LOC_Os10g20830.1 & OsHyPRP29 & 23 & $S$ & 114 & 11.75 & 6.44 \\
\hline LOC_Os10g20840.1 & OsHyPRP30 & 23 & $S$ & 114 & 11.82 & 6.86 \\
\hline LOC_Os10g20860.1 & OsHyPRP31 & 23 & $S$ & 110 & 11.51 & 5.98 \\
\hline LOC_Os10g20890.1 & OsHyPRP32 & 28 & $S$ & 98 & 10.17 & 7.08 \\
\hline LOC_Os10g40420.1 & OsHyPRP33 & 34 & $\mathrm{~S}$ & 128 & 12.58 & 8.68 \\
\hline LOC_Os10g40430.1 & OsHyPRP34 & 23 & $S$ & 123 & 12.27 & 7.07 \\
\hline LOC_Os10g40440.1 & OsHyPRP35 & 23 & $S$ & 119 & 12.05 & 6.62 \\
\hline LOC_Os10g40460.1 & OsHyPRP36 & 25 & $S$ & 106 & 10.96 & 6.85 \\
\hline LOC_Os10g40470.1 & OsHyPRP37 & 24 & $S$ & 107 & 10.99 & 6.92 \\
\hline LOC_Os10g40480.1 & OsHyPRP38 & 24 & $S$ & 112 & 11.58 & 7.72 \\
\hline LOC_Os10g40510.1 & OsHyPRP39 & 21 & $S$ & 112 & 11.49 & 7.38 \\
\hline LOC_Os10g40520.1 & OsHyPRP40 & 22 & $S$ & 100 & 10.42 & 8.00 \\
\hline LOC_Os10g40530.1 & OsHyPRP41 & 21 & $\mathrm{~S}$ & 111 & 11.55 & 8.05 \\
\hline LOC_Os10g40614.1 & OsHyPRP42 & 26 & $\mathrm{~S}$ & 141 & 13.17 & 7.91 \\
\hline LOC_Os11g02165.1 & OsHyPRP43 & 29 & $\mathrm{C}$ & 222 & 22.95 & 9.45 \\
\hline LOC_Os12g02105.1 & OsHyPRP44 & 28 & $\mathrm{C}$ & 219 & 22.43 & 9.35 \\
\hline LOC_Os12g28880.1 & OsHyPRP45 & 24 & $\mathrm{C}$ & 148 & 15.28 & 8.74 \\
\hline LOC_Os12g29040.1 & OsHyPRP46 & 20 & M & 190 & 20.01 & 8.26 \\
\hline
\end{tabular}

a Subcellular target for each protein. S: secretory pathway; M: mitochondria: C: chloroplast. 


\subsection{Chromosomal Location, Gene Duplication and Structural Analysis of OsHyPRPs}

The mapping of 46 OsHyPRP genes on 12 rice linkage groups (LGs) revealed their biased distribution (Figure 2). Out of 46 OsHyPRP genes, 35 genes ( $>75 \%)$ are present on LG3, LG4, LG6, and LG10 with 8, 5, 5, and 17 OsHyPRP genes, respectively, while the LG1, LG5, LG8, and LG9 are devoid of OsHyPRPs (Figure 2). The gene cluster analysis showed that 31 OsHyPRP genes $(>67 \%)$ are present in nine clusters, distributed on six LGs with the largest gene cluster of 10 genes on LG10. The gene duplication analysis of OsHyPRP genes revealed the presence of $11.1 \%, 20 \%, 8.8 \%$, and $62 \%$ of singleton, dispersed, proximal, and tandem duplication, respectively. Of the total 46 OsHyPRP genes, 21 are tandemly duplicated (Supplementary Figures S1 and S2), this indicates that ongoing tandem duplications in the rice genome $[12,45]$ might be causing OsHyPRP gene family expansion in rice. Results from the gene structure analysis of OsHyPRP genes showed that the majority of them (33 genes, $71 \%$ ) are intron-less, while the remaining genes (13 genes, $28 \%$ ) have introns that vary from 1 to 3 in number (Supplementary Figure S3).

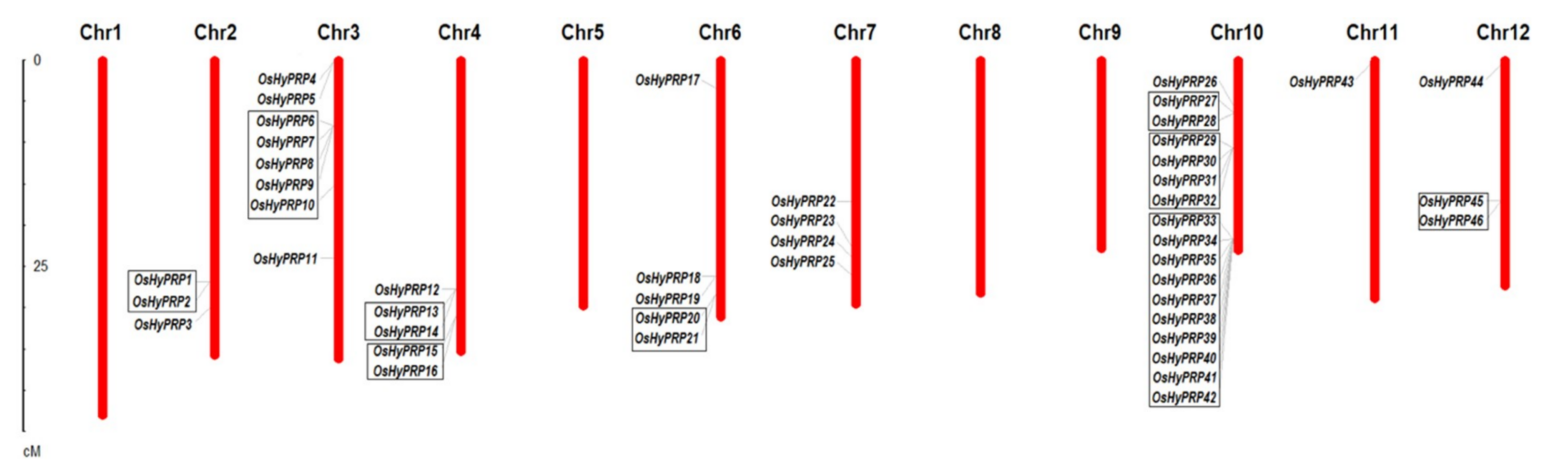

Figure 2. Chromosomal mapping and clustering of 46 OsHyPRP gene models in rice chromosomes. The boxes indicate the genes present within the region of $200 \mathrm{Kbp}$ to form a gene cluster.

\subsection{Phylogenetic Analysis of the OsHyPRP Family among Different Plant Species}

To analyze the phylogenetic relationships of the rice OsHyPRPs with that of HyPRPs of other plant species, a total of 144 HyPRP proteins; 46 of rice, 24 of Arabidopsis, 30 of maize, 34 of sorghum, and 10 of Brachypodium distachyon were used to construct a maximum-likelihood phylogenetic tree with 500 bootstrap replicates. The phylogenetic tree categorized all the $144 \mathrm{HyPRP}$ proteins into seven distinct clades (A to G; Figure 3). Notably, the majority of OsHyPRPs were found to cluster together in separate sub-clades along with the HyPRPs of maize and sorghum in clade B and F (19 and 12 OsHyPRPs, respectively), while the Arabidopsis HyPRPs were mainly present in clade A and C (Figure 3). The clustering of the HyPRPs of rice, maize, and sorghum together, and separation from Arabidopsis HyPRPs may indicate their early divergence during monocotyledon lineage-specific evolution. The random distributions of B. distachyon HyPRPs in several clades offers an exception to this hypothesis, which may be due to high sequence diversity and low numbers of HyPRPs in B. distachyon. The phylogenetic analysis also revealed that the majority of OsHyPRPs clustered together into sub-clades are also tandemly duplicated and found to present as gene clusters on rice LGs (Figures 2 and 3, and Supplementary Figure S2). 


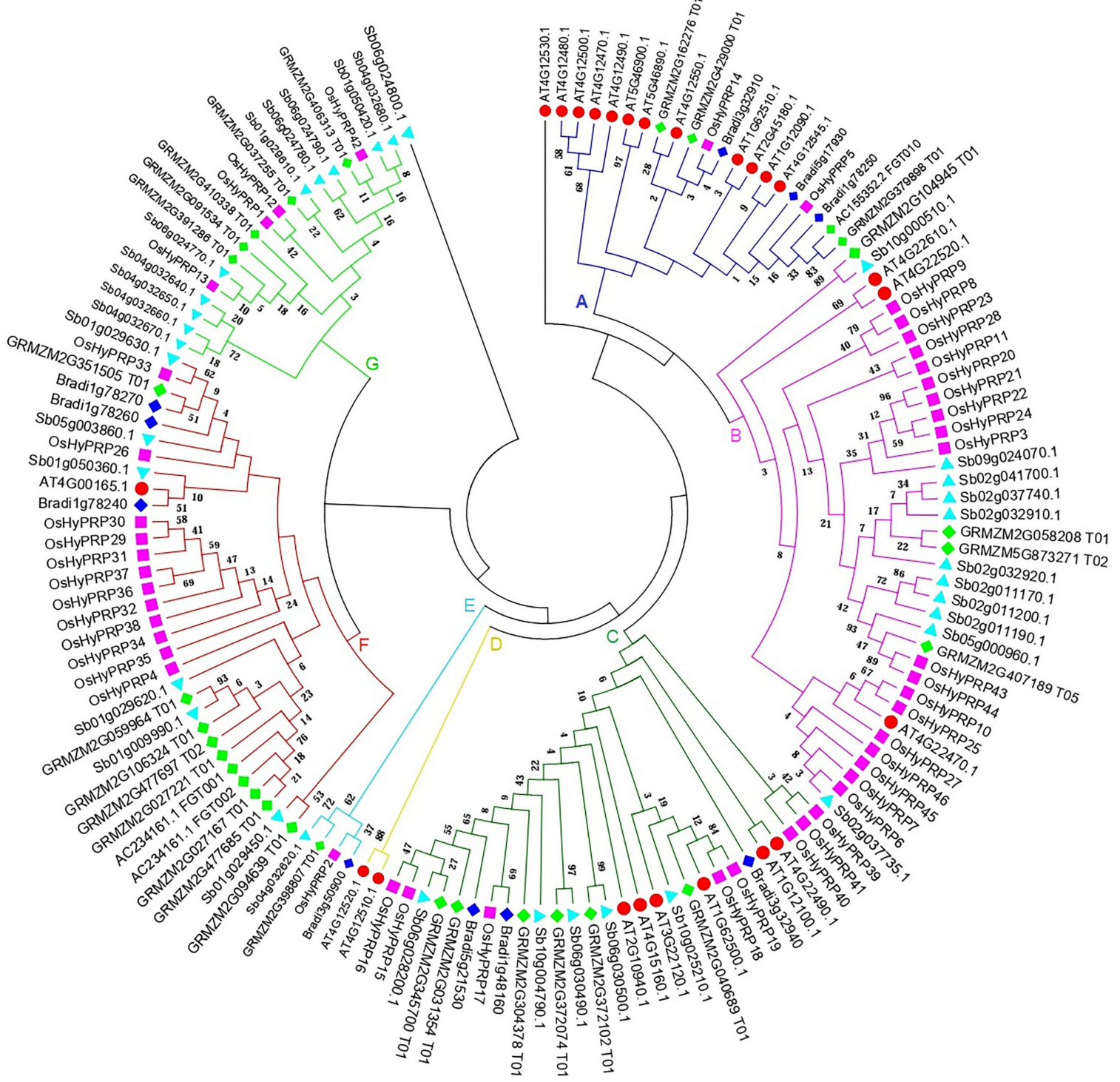

Figure 3. Phylogenetic relationship of rice HyPRPs with that of Arabidopsis, maize, sorghum and B. distachyon. The $8 \mathrm{CM}$ region of all the HyPRPs was used to construct the phylogenetic tree. The multiple sequence alignment and phylogenetic tree construction were performed with MEGA6.06 using maximum likelihood method with 500 bootstrap replicates. The different shapes with color code represent the HyPRPs of different plant species; red sphere for Arabidopsis, magenta square for rice, cyan triangle for sorghum, lawn-green diamond for maize, and blue diamond for Brachypodium. The one protein each from sorghum and Arabidopsis does not group with other HyPRPs and thus considered as highly divergent outliers.

\subsection{OsHyPRPs Expression Profile in Response to M. oryzae}

Under natural environmental conditions, plants are vulnerable to various biotic and abiotic stresses that severely affect their productivity. Sometimes, the exposure to abiotic stresses compromises the plant's resistance toward biotic stress through reorientation of plant-pest interaction [46,47]. Moreover, the biotic agent directly uses the plant resources, which eventually leads to reduced plant vigor [48]. Therefore, it is of great significance to identify the key regulators that drive the defense response of plants towards biotic and abiotic stresses. The rice blast disease is a major risk to sustainable rice production since it causes 10-30\% of global loss annually [49]. In one of the previous studies from our group, we performed the RNA-Seq based transcriptome analysis for $M$. oryzae inoculated rice 
blast susceptible PB1 and blast-resistant PB1+Pi9 rice lines at $24 \mathrm{~h}$ post-infection (hpi) [43]. This data is available at the Gene Expression Omnibus (GEO) dataset with accession number GSE81906 (www.ncbi.nlm.nih.gov/gds/?term=GSE81906[Accession]). Using this data, we analyzed the expression of OsHyPRPs, where out of $46 \mathrm{OsHyPRPs}$ genes 15 were found to exhibit expression in at least one of the samples, while the rest of the 31 genes showed no expression. A higher number of OsHyPRP genes were found to have up-regulated expression in PB1+Pi9 when compared to PB1, after 24 hpi with $M$. oryzae (Figure 4A). Based on the transcriptome analysis, we selected six highly-expressed OsHyPRP genes (OsHyPRP5, OsHyPRP14, OsHyPRP15, OsHyPRP16, OsHyPRP39, and OsHyPRP40) for their qRT-PCR based expression profiling in response to biotic \& abiotic stresses, and hormone treatment. Out of the six selected OsHyPRP genes, we observed the qRT-PCR based expression of four OsHyPRP (OsHyPRP5, OsHyPRP14, OsHyPRP16, and OsHyPRP40) genes.

The qRT-PCR analysis of collected samples was performed only after the appearance of disease lesions at seven days post-inoculation of M. oryzae in PB1 (Figure 4B). The expression of all the four OsHyPRPs showed induction in response to M. oryzae in PB1+Pi9 (Figure 4C). However, the expression of OsHyPRP5 was early inducible, a similar expression pattern was observed in both PB1+Pi9 and PB1 upon M. oryzae inoculation (Figure 4C). In addition to OsHyPRP5, OsHyPRP14 and OsHyPRP16 also exhibited early induced expression (>three-fold up-regulation) at $12 \mathrm{hpi}$ in response to $M$. oryzae inoculation (Figure 4C). Comparatively, among the other genes, the expression of OsHyPRP16 was strongly induced upon M. oryzae inoculation in PB1+Pi9. In the blast-susceptible PB1 rice line, the expression of OsHyPRP14, OsHyPRP16, and OsHyPRP40 either remained at the basal level or down-regulated in response to $M$. oryzae, except for 2.8-fold up-regulation of OsHyPRP14 at 12 hpi (Figure 4C). This finding shows that the presence of Pi9 positively regulates the expression of OsHyPRPs, and this induced expression may be involved in the enhanced defense response in rice blast resistant PB1+Pi9 plants. Additionally, our qRT-PCR based expression patterns of OsHyPRPs are comparable with that of the RNA-Seq transcriptome data of Jain et al. [43] (Figure 4A,C) and thus further corroborate the up-regulation of OsHyPRP genes in PB1+Pi9 as compared to PB1, upon M. oryzae inoculation.

Similar to the present study, the early induced up-regulation of Arabidopsis AtDHyPRP1 has been observed in response to P. syringae [28]. Likewise, the C. gloeosporioides infected walnut was found to show early induced expression of the JsPRP1 gene [24]. In soybean, the expression of GmHyPRPs and SbPRP was found to be up-regulated in response to Phakopsora pachyrhizi [27] and soybean mosaic virus [26], respectively. Several studies have reported the role of HyPRPS to confer the disease resistance towards different plant pathogens. Recently, Liu et al. [24] found that the overexpression of the walnut HyPRP gene, JsPRP, in tobacco enhances the resistance against C. gloeosporioides in transgenic plants. Additionally, using in vitro assay, the recombinant JsPRP protein was found to show antifungal activity towards different fungal pathogens such as C. gloeosporioides, Gibberella moniliformis, Botryosphaeria dothidea, and Fusarium solani [24]. The similar antifungal activity of Arabidopsis HyPRP protein, EARLI1, against Saccharomyces cerevisiae has been reported by Li et al. [50]. Additionally, the exogenous application of recombinant EARLI1 was found to inhibit the conidial germination and hyphae growth of Botrytis cinerea and Fusarium oxysporum [50]. In a recent study, the CcHyPRP overexpressing transgenic rice lines exhibit enhanced tolerance towards both biotic and abiotic stresses, where enhanced tolerance towards biotic stresses was suggested to be plausibly contributed by the higher endochitinase activity in transgenic rice plants [37]. Jung et al. [38] illustrated the role of EARLI-type HyPRP, AZI1, as a component of plant systemic immunity, where AZI1 may act to regulate and/or directly translocate the mobile signals during SAR in response to biotic stress. All these findings suggest that the HyPRPS perform a diverse role in enhancing plant tolerance towards different biotic stresses. 
A

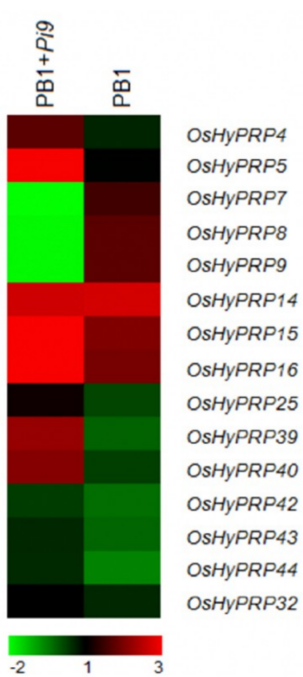

C

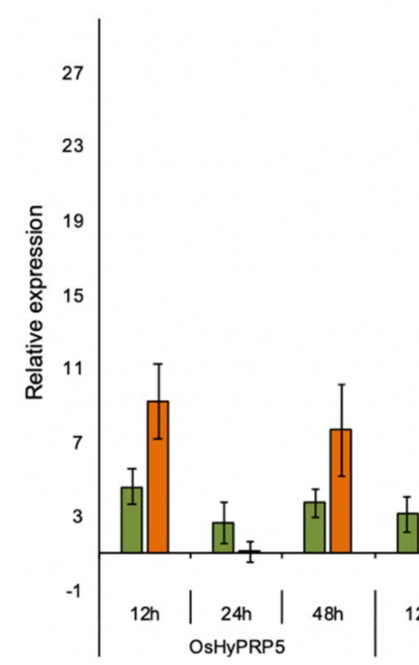

B

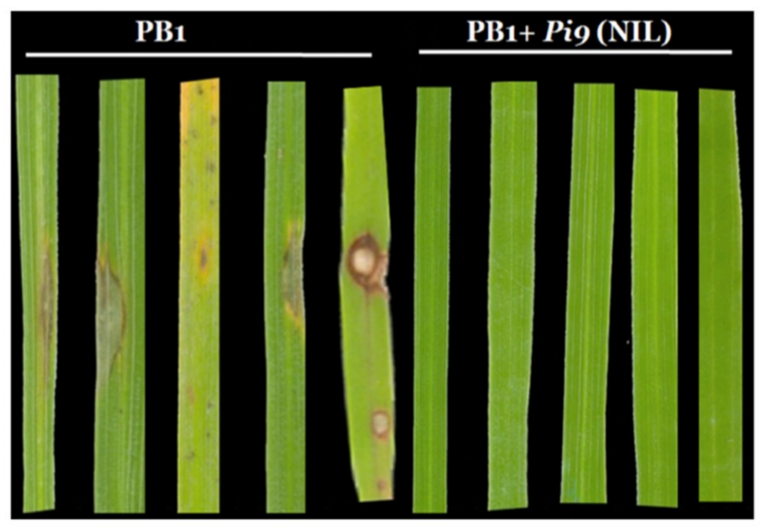

M. oryzae infection

$\square \mathrm{PB} 1+\mathrm{Pi}$ 口PB1

Figure 4. The expression of OsHyPPR genes in response to M. oryzae. (A) The heat map for the expression profile of OsHyPRP genes as determined through RNA-Seq analysis of PB1 and PB1+Pi9 in response to $M$. oryzae after $24 \mathrm{hpi}$. The expression shown here is the log2 fold change value as compared to mock-treated PB1 and PB1+Pi9. (B) The picture is showing disease lesions in PB1 after seven days of M. oryzae inoculation, while no disease lesions were present in PB1+Pi9 (C) The qRT-PCR based expression profile of four OsHyPRP genes showed up-regulated expression in RNA-Seq analysis. The calculated expression level was relative to the untreated respective rice lines. The experiment was performed with three technical replicates of three independent biological replicates. The error bars represent the standard error of the means of three independent biological replicates. The rice elongation factor-1A (OsEF-1a) gene was used to normalize the qRT-PCR expression data.

In addition, a few other studies have also reported negative effects of $H y P R P S$ on plant's resistance against biotic stress. In one recent study, using a gain- and loss-of-function approach, Yang et al. [40] suggested the role of GbHyPRP1 as a negative regulator of disease resistance in cotton against pathogenic fungi Verticillium dahlia. Using a similar approach, Yoem et al., [39] characterized two HyPRP genes; CaHyPRP1 from C. annuum (pepper) and NbHyPRP1 from Nicotiana benthamiana. The transient overexpression of CaHyPRP1 in N. benthamiana enhances the disease susceptibility towards virulent P. syringae pv. tabaci. In contrast, the silencing of CaHyPRP1 and NbHyPRP1 resulted in the enhanced basal defense against virulent and avirulent plant pathogens through suppression of pathogen-induced cell death [39]. Based on these results, Yoem et al. [39] suggested that CaHyPRP1 and NbHyPRP1 perform the dual function as a positive regulator of cell death and negative regulator of plant basal 
defense against pathogens. Together, in the light of previous findings, the early and strong induction of OsHyPRPs, particularly OsHyPRP14 and OsHyPRP16 genes in PB1+Pi9 suggests that these genes may contribute towards the enhanced tolerance against $M$. oryzae in the blast-resistance PB1+Pi9 rice line.

\subsection{OsHyPRPs Expression Profile under Abiotic Stress}

Abiotic factors such as salt, drought, and temperature are the key environmental cues which directly affect the plant's geographical distribution, physiology, and productivity $[47,48,51-56]$. In the present investigation, the expression profiling of OsHyPRP genes under salt, cold, and heat stress revealed their differential expression. In $\mathrm{PB} 1+P i 9$, under salt stress, all the genes exhibit induced expression, except for OsHyPRP5 that remains at the basal level at all the time points (Figure 5A). Among the early induced OsHyPRPs, the OsHyPRP14 exhibit more than seven-fold up-regulation at $12 \mathrm{~h}$ of salt treatment, which reduced to the basal level at subsequent time points (Figure 5A). Although the OsHyPRP16 shows no early induction, its expression was more than three-fold up-regulated at $24 \mathrm{~h}$ and $48 \mathrm{~h}$ of salt treatment in PB1+Pi9 (Figure 5A). In the case of PB1, only the OsHyPRP5 exhibit increased expression in response to salt stress, while the other genes remain either at the basal level or down-regulated as compared to untreated control (Figure 5A).

A

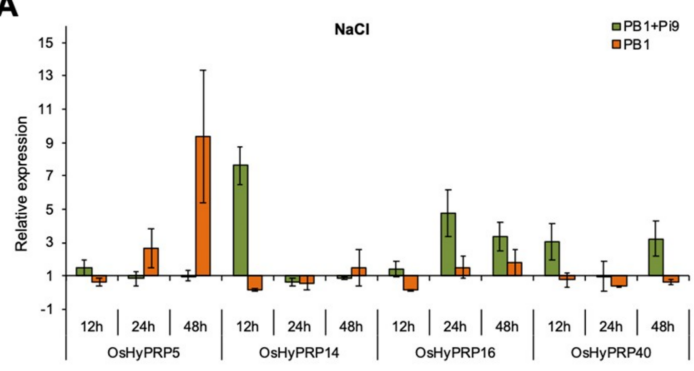

B

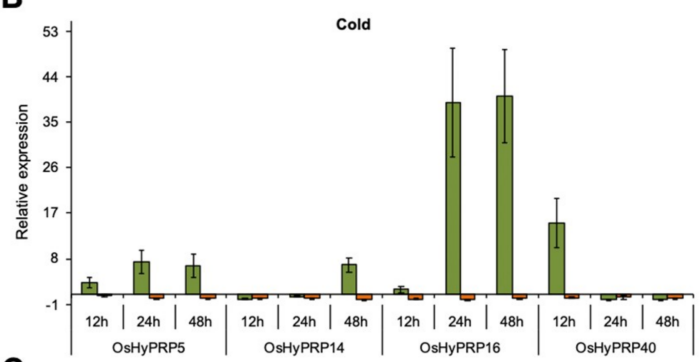

C

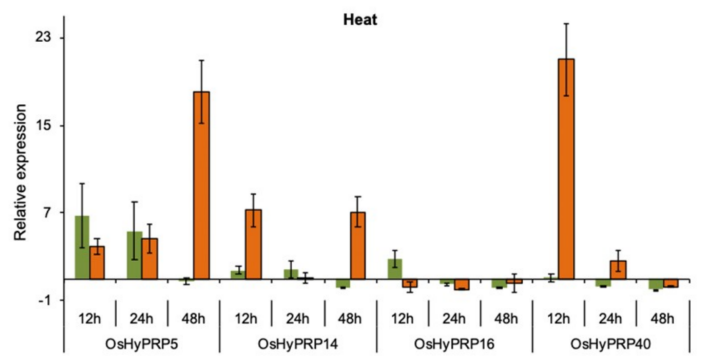

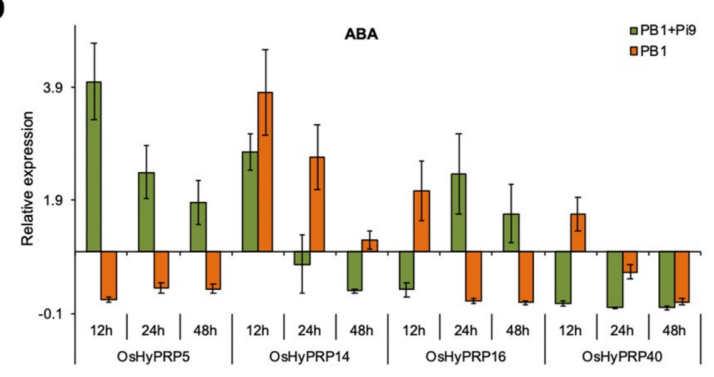

E
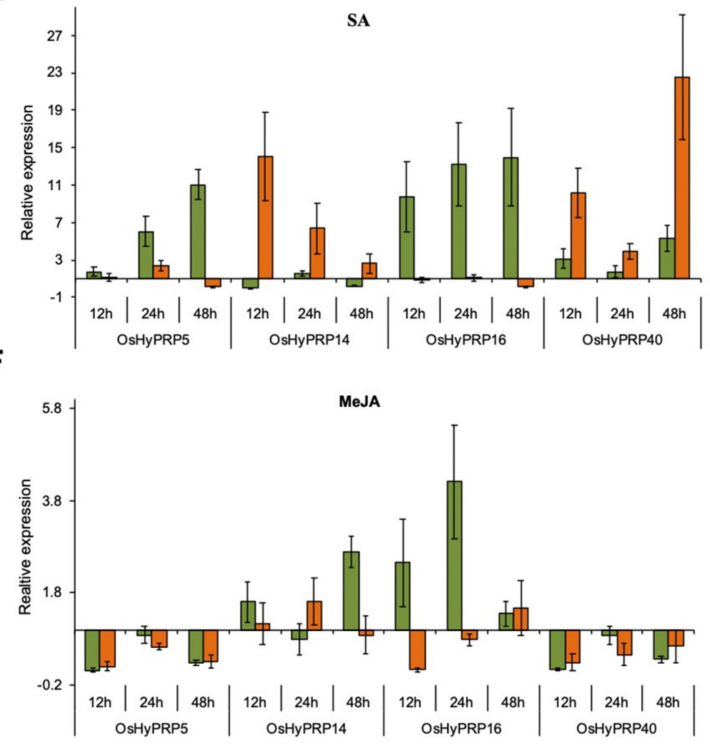

Figure 5. The qRT-PCR based expression of four selected OsHyPPR genes in PB1 and PB1+Pi9, in response to stress such as $\mathrm{NaCl}(\mathbf{A})$, cold (B), and heat (C), and hormone treatments including $\mathrm{ABA}(\mathbf{D})$, SA (E) and MeJA (F). The calculated expression level was relative to the untreated rice seedlings of respective rice lines. The experiment was performed with three technical replicates of three independent biological replicates. The error bars represent the standard error of the means of three independent biological replicates. The rice elongation factor-1A (OsEF-1a) gene was used to normalize the qRT-PCR expression data. 
Surprisingly, we found that all four OsHyPRP genes were down-regulated in PB1 under cold stress. In contrast to PB1, the OsHyPRP5 and OsHyPRP16 exhibit early induced and consistently up-regulated expression in PB1+Pi9 under cold stress, whereas OsHyPRP16 shows comparatively strong up-regulation ( $>39$-fold at $24 \mathrm{~h}$ and $48 \mathrm{~h}$ ) (Figure 5B). Although the OsHyPRP14 and OsHyPRP40 were also found to be cold-inducible, their expression shows up-regulation only at $48 \mathrm{~h}$ (6.8-fold) and $12 \mathrm{~h}$ (15.1-fold), respectively (Figure 5B). In contrast to cold stress, the majority of OsHyPRPs exhibits up-regulation in PB1 under heat stress, except for OsHyPRP16, whose expression remains constantly down-regulated (Figure 5C). In PB1, the expression of OsHyPRP5 showed a continuous increase (four-fold at $12 \mathrm{~h}$ to 18 -fold at $48 \mathrm{~h}$ ) in response to heat stress, while in PB1+Pi9 it showed a reciprocal pattern of expression (6.7-fold at $12 \mathrm{~h}$ to 0.7 -fold at $48 \mathrm{~h}$ ) (Figure 5C). Besides OsHyPRP5, the OsHyPRP14 (>seven-fold at $12 \mathrm{~h}$ and $48 \mathrm{~h}$ ) and OsHyPRP40 (21-fold at $12 \mathrm{~h}$ ) also exhibit up-regulation in PB1 under heat stress, while in contrast, their expression was remained either at the basal level or down-regulated in PB1+Pi9 (Figure 5C). In PB1+Pi9, the OsHyPRP16 was the only gene that showed differentially up-regulated expression (2.8-fold) at $12 \mathrm{~h}$ of heat stress, as compared to PB1 (Figure 5C).

In summary, the expression of OsHyPRP14, OsHyPRP16, and OsHyPRP40 showed differential up-regulation in PB1+Pi9, as compared to PB1, during all the abiotic stresses (Figure 5A-C). Similar to the present study, the expression of pigeonpea $C c H y P R P$ showed strong induction in the leaf and root tissues in response to different abiotic stresses such as drought, salt, heat, and cold $[20,57]$. In cotton, the strongly up-regulated expression of GhHyPRP4 in cold stress, and GhHyPRP3 in several abiotic stresses such as dehydration, cold and salt stress has been observed [21,22]. Likewise, the cold-inducible expression of Arabidopsis HyPRPs, namely EARLI1 and AZI1, has been reported [58-60], where the $A Z I 1$ was also observed to show salt stress-inducible expression [61]. Similarly, in response to cold and salt stress, the P. trifoliata PtrPRP exhibits induced expression [36]. The expression of msa CIC (HyPRP gene) in cold-tolerant $M$. sativa [16] and, MfHyPRP in M. falcata was found to be cold-inducible [23]. Furthermore, the $M f H y P R P$ was found to show an inducible expression in response to drought stress and, hydrogen peroxide and nitric oxide treatment; however, its expression remains low under salt stress [23]. Similarly, the soybean SbHyPRP was found to show inducible expression only under low salt conditions, while its expression remained low under high salt conditions [26].

Several functional studies have been performed in the last two decades to characterize the role of HyPRPs in response to abiotic stresses. The EARLI-type HyPRPs in Arabidopsis were found to play a significant role in cold and salt stress tolerance $[19,59,60]$. The RNA interference (RNAi) based suppression of EARLI resulted in the reduced freezing tolerance in EARLI suppressed Arabidopsis lines [19]. Moreover, higher electrolyte leakage in the EARLI1 knockdown Arabidopsis, as compared to EARLI1 overexpressing Arabidopsis, suggests that EARLI1 enhances the freezing tolerance through reduction of freezing-induced cellular damage [35]. Additionally, the overexpression of EARLI1 in Arabidopsis plants conferred enhanced tolerance towards salt stress [59]. Similar to EARLI1, its homolog in Arabidopsis, AZI1, was also found to enhance the salt and freezing tolerance in AZI1 overexpressing Arabidopsis $[60,61]$. The AZI1 was found to act as a direct target of mitogen-activated protein kinase MPK3, where the presence of MPK3 is essential for strong and robust tolerance towards salt stress [61]. The overexpression of MfHyPRP in tobacco conferred enhanced tolerance towards freezing and chilling stress, in addition to the methyl viologen induced oxidative stress [23]. Similarly, the ectopic expression of $C c H y P R P$ in yeast, Arabidopsis, and rice resulted in the enhanced tolerance towards multiple abiotic stresses, including drought, salt, and heat stresses [20,37]. Moreover, under abiotic stress conditions, the transgenic rice overexpressing $C c H y P R P$ showed a higher survival rate with more productivity when compared to wild type [37]. Using the RNAi approach, Peng et al. [36] showed that the PtrPRP suppressed P. trifoliate plants were more susceptible to cold stress than wild type plants, which indicates the role of $\operatorname{PrPRP}$ as a positive regulator of cold tolerance. In contrast, Li et al. [29] demonstrated the negative role of tomato SpHyPRP1 on abiotic stresses tolerance, where SPHyPRP1 was found to be responsible for the reduced ROS-scavenging and thus compromised abiotic stress tolerance, which is believed to be contributed by ROS-scavenging [62]. All the above previous 
findings suggest that HyPRPs are involved either as a positive regulator $[20,37,59,60]$ or negative regulator $[29,39]$ of plants stress tolerance. In the present study, the strongly induced expression of OsHyPRP16 and OsHyPRP40 was found to be more consistent at least during cold and salt stresses, which indicates their possible role in defense response towards these abiotic stresses.

\subsection{Expression Profiling of OsHyPRPs under Phytohormone Treatment}

Phytohormones are small signaling molecules that perform diverse functions such as regulation of cellular and developmental processes during the plant lifespan [63-65]. The accumulation of phytohormones such as ABA, SA, and JA (Jasmonic acid), and their cross-talk accompanied the stress tolerance in plants [66-70]. Since the hormones signaling pathways are crucial for biotic and abiotic stress responses, we performed the expression profiling for selected OsHyPRP genes in response to stress-related hormones namely, ABA, SA, and MeJA.

In ABA-treated PB1+Pi9, the OsHyPRP5 (four-fold) and OsHyPRP14 (>2.7-fold) exhibit early induced up-regulated expression at $12 \mathrm{~h}$ (Figure 5D). The expression of OsHyPRP16 was found to show up-regulation (2.4-fold) only at $24 \mathrm{~h}$ in PB1+Pi9. Notably the expression of OsHyPRP14 and OsHyPRP16 follow the similar temporal expression pattern in PB1+Pi9 under salt stress and ABA treatments (Figure 5A,D). Unlike others, the expression of OsHyPRP40 constantly remained down-regulated in PB1+Pi9 (Figure 5D). In the case of ABA-treated PB1, the expression of OsHyPRP5 constantly remained down-regulated (Figure 5D). The OsHyPRP14 follows a similar decreasing expression pattern in both PB1+Pi9 and PB1, with a comparatively abrupt decrease in the case of PB1+Pi9 (Figure 5D). The expression of OsHyPRP16 and OsHyPRP40 was found to show up-regulation only at $12 \mathrm{~h}$ of ABA treatment in PB1 (Figure 5D). In summary, the ABA treatment resulted in the differential and induced expression of OsHyPRP5 and OsHyPRP16 in PB1+Pi9, as compared to PB1. Similar to our study, the ABA treatment resulted in a strong and early induced expression of $C c H y P R P 1$ in pigeonpea roots, MfHyPRP in M. falcate, and PtrPRP in P. trifoliata [20,23,36]. In contrast, the BnPRP in B. napus, GhHyPRP4 in cotton and SbPRP in soybean, and SpHyPRP1 in tomato were found to be down-regulated upon ABA treatment $[18,21,26,29]$. Since the suppression of negative regulators or overexpression of positive regulators of $\mathrm{ABA}$ response is known to confer drought tolerance [19,71], the down-regulation of $S p H y P R P 1$ in response to exogenous ABA, and the reduced growth along with enhanced salt and drought sensitivity in SPHYPRP1 overexpressing transgenic tomato suggest the negative role of SpHyPRP1 in ABA signaling [29]. In the present analysis, the OsHyPRP5 and OsHyPRP16 were differentially induced in ABA-treated PB1+Pi9 as compared to PB1 (Figure 5D), which indicates the positive effect of Pi9 on their expression. However, it is required to validate the role of OsHyPRP5 and OsHyPRP16 in ABA-mediated salt and drought stress response.

In response to SA treatment, the OsHyPRP5 and OsHyPRP16 exhibit up-regulated expression in $\mathrm{PB} 1+P i 9$, while their expression remains either at the basal level or down-regulated in PB1, except for the 2.4-fold up-regulation of OsHyPRP5 at $24 \mathrm{~h}$ (Figure 5E). Although the OsHyPRP40 also exhibit up-regulated expression in response to SA, it shows a similar expression pattern in both $\mathrm{PB} 1+\mathrm{Pi} 9$ and PB1, with comparatively strong up-regulation in the latter (Figure 5E). Unlike other genes, the expression of OHYPRP14 was only up-regulated in PB1 upon SA treatment, while it remained either at the basal level or down-regulated in PB1+Pi9 (Figure 5E). Similar to ABA treatment, the expression of OsHyPRP5 and OsHyPRP16 was also induced in SA-treated PB1+Pi9 (Figure 5D,E). In MeJA-treated $\mathrm{PB} 1+\mathrm{Pi9}$, only the OsHyPRP16 exhibit early and strong induction (2.5-fold at $12 \mathrm{~h}$ ) with peak expression at $24 \mathrm{~h}$ (4.2-fold) (Figure 5F). However, the expression of OsHyPRP14 also showed up-regulation (2.7-fold at $48 \mathrm{~h}$ ) in the later stages of MeJA treatment in PB1+Pi9 (Figure 5F). Meanwhile, in PB1, the expression of OsHyPRP14 and OsHyPRP16 remains either at the basal level or down-regulated in response to MeJA (Figure 5F). Unlike other hormone treatments, the expression of OsHyPRP5 and OsHyPRP40 remains low in both MeJA-treated rice lines (Figure 5F). Altogether, in response to each ABA, SA, and MeJA treatment in PB1+Pi9, the OsHyPRP16 is the only gene that showed up-regulated expression, at least in one of the time points of hormone treatment. 
Only a few previous studies are available that have analyzed the expression profile of HyPRPs in response to SA and MeJA/JA treatments. In soybean, the expression of SbPRP was found to show rapid induction upon SA treatment, while in contrast, its expression shows down-regulation in response to MeJA treatment [26]. In the present investigation, the OsHyPRP5 and OsHyPRP40 showed a similar expression behavior in response to SA and MeJA treatment in PB1+Pi9 (Figure 5E, F). Li et al., [28] reported the induced expression of AtDHyPRP1 in response to MeJA and SA treatment as well as in response to $P$. syringae. We also observed the induced expression of OsHyPRP16 under biotic stress and hormone treatments, including ABA, SA, and MeJA (Figure 5D-F). In a recent study, the expression of cotton $G b H y P R P$ was strongly down-regulated in response to SA treatment, while it was significantly up-regulated in each ABA, JA, and ethylene treatment [40]. Based on the similarity in the expression profile of GbHyPRP in response to SA and V. dahlia inoculation, Yang et al. [40] proposed a negative role of GbHyPRP towards $V$. dahlia resistance in cotton via a SA-mediated signaling pathway [40]. Intriguingly, in the present study, the up-regulation of OsHyPRP16 also follows a similar expression pattern in SA-treated and M. oryzae inoculated PB1+Pi9 (Figures 4 and 5E) [41,42]. Based on this expression similarity, we also suspect that OsHyPRP16 and SA-mediated signaling might be involved to confer blast-resistance in PB1+Pi9, where Pi9 is supposed to have a central role in activating the basal defense pathway.

\subsection{Analysis of Cis-Regulatory Elements in Four OsHyPRP Promoter Sequences}

The cis-elements act as molecular switches that participate in the transcriptional regulation of genes during different environmental cues [72]. The $1.5 \mathrm{~kb}$ upstream DNA sequences of the four selected OsHyPRP genes were retrieved and analyzed using the PLACE database. The list of various putative cis-regulatory elements present in the upstream sequences is given in Supplementary Table S1. The promoter sequences were found to contain sequence motifs that are responsive to biotic stresses (pathogen, elicitor, and disease resistance responsive elements), abiotic stress (cold, heat, salt, drought, and wounding responsive elements) and hormones (ABA, SA, JA, and GA responsive elements) (Figure 6 and Supplementary Table S1). The detailed analysis of sequence motifs revealed that the promoter sequence of OsHyPRP16 and OsHyPRP40 contains the highest number of sequence motifs that are responsive to biotic and abiotic stresses and, hormones treatment. However, the expression of OsHyPRP40 under different experimental conditions did not show correlation with the number of cis-regulatory elements in its promoter sequence, except for $M$. oryzae inoculation and SA treatment (Figures $4 \mathrm{C}$ and $5 \mathrm{E}$ and Supplementary Table S1). Similarly, in one of the previous studies, besides the absence of cold-responsive elements, the GhHyPRP4 promoter was found to be cold-inducible as shown by the high activity of GhHyPRP4 promoter-driven $\beta$-glucuronidase (GUS) gene in transgenic Arabidopsis under cold stress [21]. In contrast to OsHyPRP40, the OsHyPRP16 exhibits a good correlation between its inducible expression in response to M. oryzae, salt, cold, ABA, SA, and $\mathrm{MeJA}$, and the occurrence of different stresses-and hormone-responsive cis-regulatory elements in the promoter sequence (Figures $4 \mathrm{C}$ and 5 and Supplementary Table S1). In particular, the promoter sequence of $\mathrm{OsHyPRP16}$ contains the highest number of cis-regulatory elements ( 28 in number) that are involved in pathogen-, elicitors-, and disease resistance-response. The early and strong inducible expression of OsHyPRP16 in PB1+Pi9, after M. oryzae inoculation (Figure 4C) and abiotic stresses (Figure 5A-C), and hormone treatment (Figure 5D-F) indicates that this gene may be actively involved in the defense-related pathways. 
OsHyPRP5

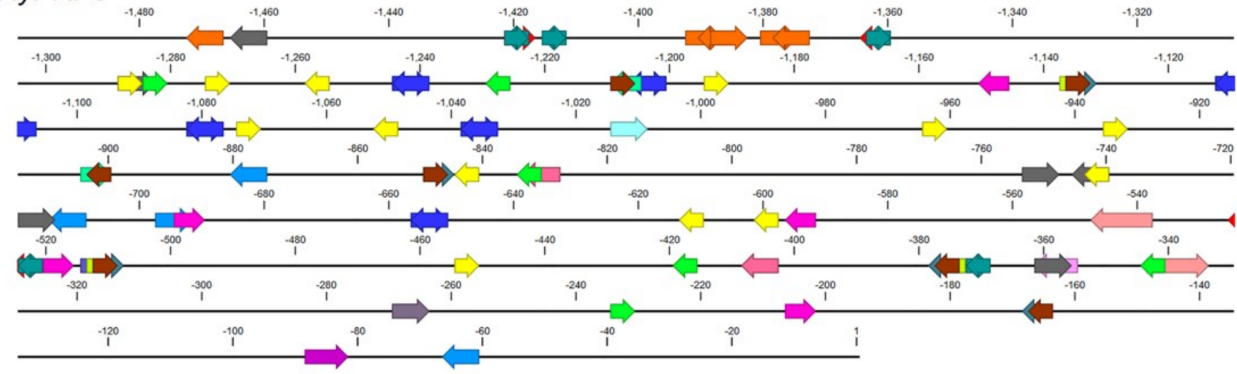

OsHyPRP14
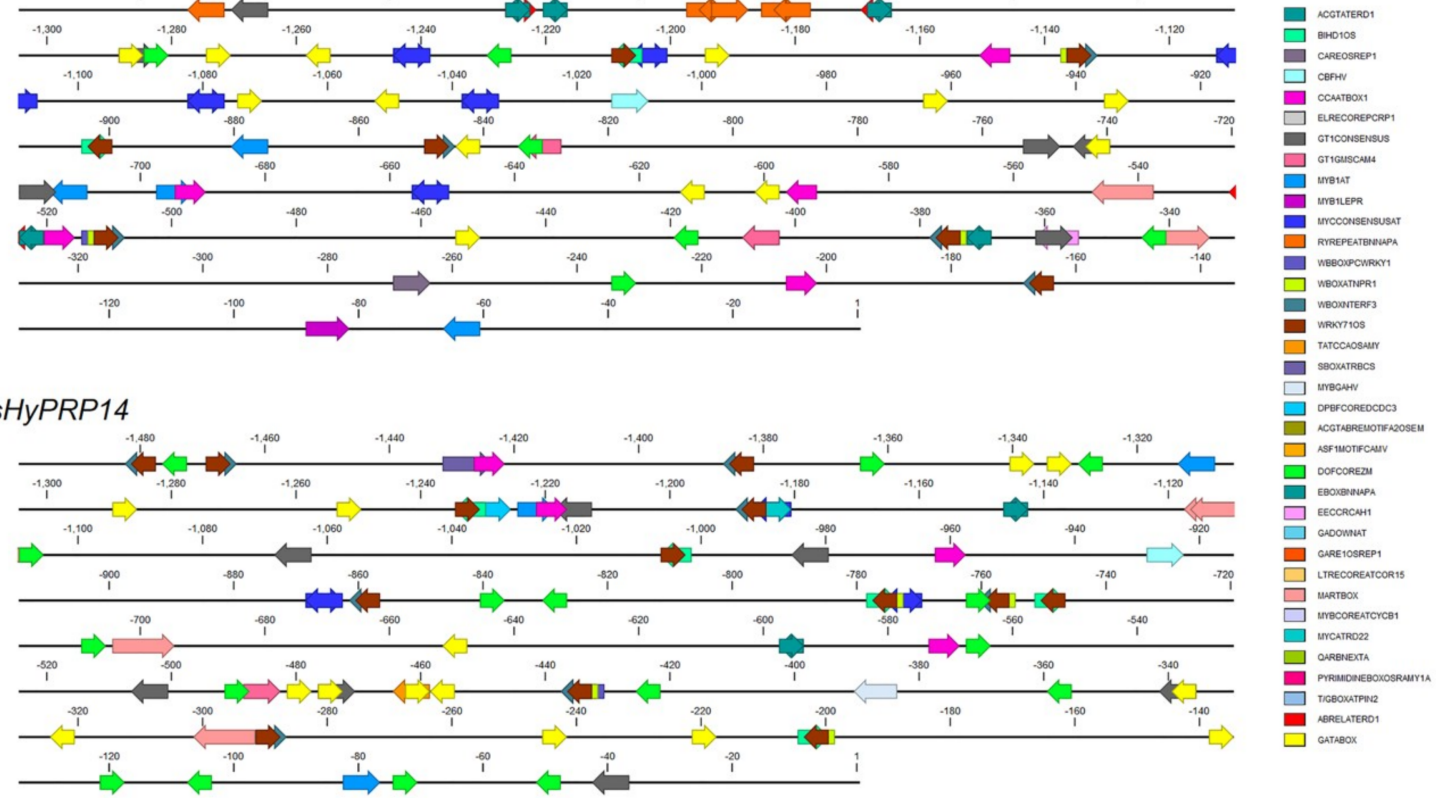

\section{OsHyPRP16}

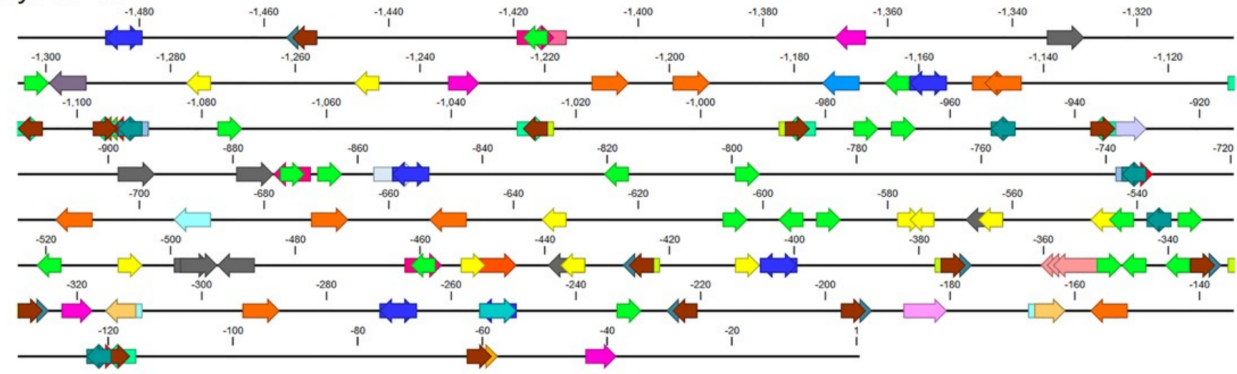

OsHyPRP40
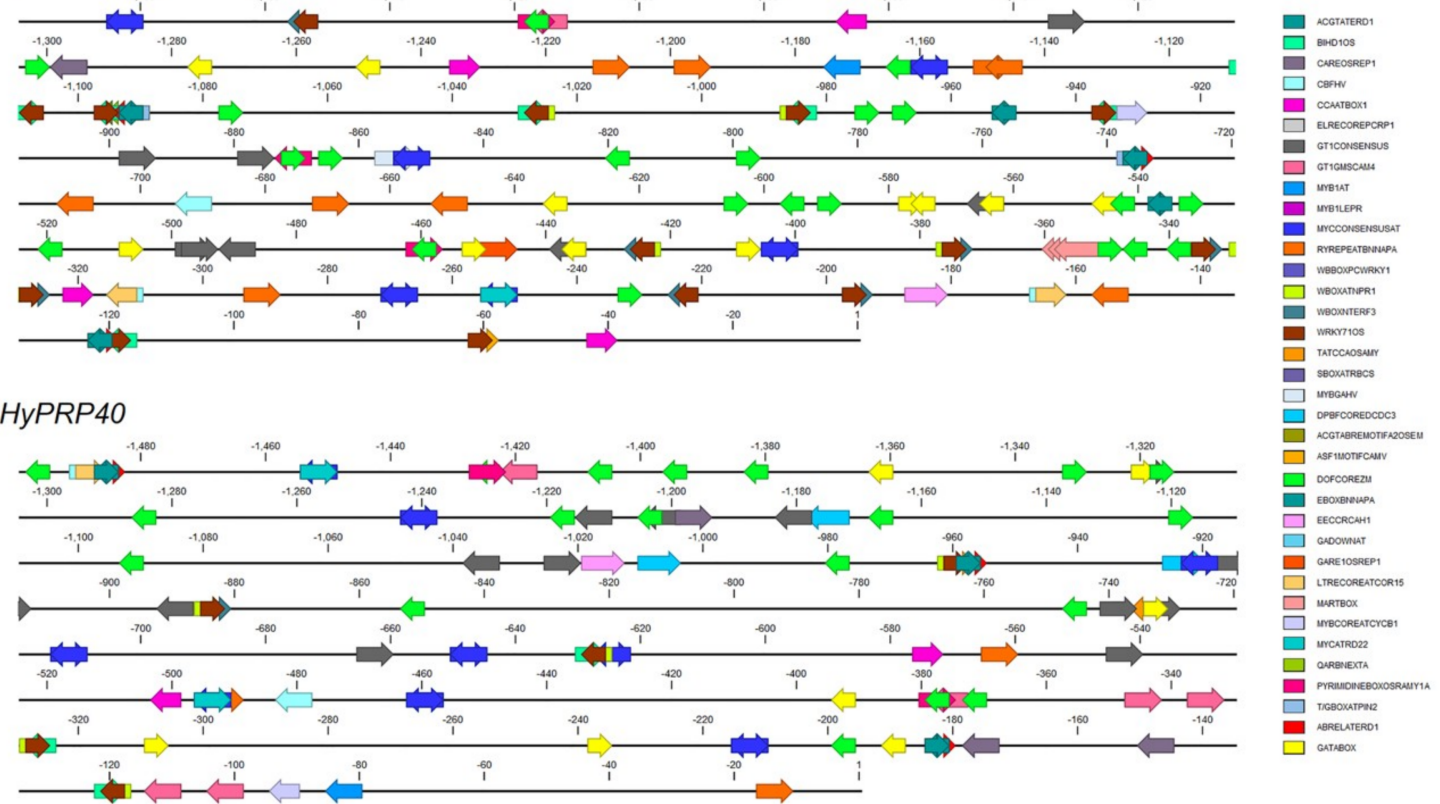

Figure 6. In silico analysis of the promoter region of four OsHyPRPs shows the presence of different cis-regulatory elements. The $1500 \mathrm{bp}$ region up-stream of ATG start codon was analyzed using the PLACE database webserver. The base immediate upstream of ATG is considered as -1 and the positions of various cis-regulatory elements are relative to -1 position. Arrows at the cis-regulatory elements indicate the position of elements on either + strand (forward direction) or - strand (reverse direction).

\subsection{Protein Structure Prediction and Docking Analysis}

The proline-rich N-terminal domain of HyPRPS suggests their role as cell-wall structural proteins; however, the expression of $H y P R P s$ in different plant tissues implies their significant roles in growth and development [9]. In HyPRPs, the presence of atypical N-terminal proline-rich domain and typical 8CM protein C-terminal domain indicate their novel functions that may be more related to $8 \mathrm{CM}$ proteins. 
The $8 \mathrm{CM}$ containing proteins, in particular, nsLTPs are known to bind hydrophobic ligands (sterols and lipids) to perform their biological functions [13-15,73]. Owing to the potential role of OsHyPRP16 in the majority of stresses, it was worthwhile to analyze the binding of OsHyPRP16 protein with different putative ligands. Previously, Yoem et al. [39] illustrated with the help of domain deletion analysis that the $8 \mathrm{CM}$ is essential for the cell death-inducing activity of CcHyPRP1. Therefore, in the present investigation, we performed in silico docking to analyze the binding of mature OsHyPRP16 (without signal peptide), and fragment deleted OsHyPRP16 (only 8CM) with that of various lipids, lipid-derivatives, fungal cell-wall components, and SA. The deduced three-dimensional (3D) structures of mature and fragment deleted OsHyPRP16 are illustrated in Figure 7. The Ramachandran plot analysis for both the predicted protein structures showed more than $90 \%$ of amino acid residues within the energetically allowed region.
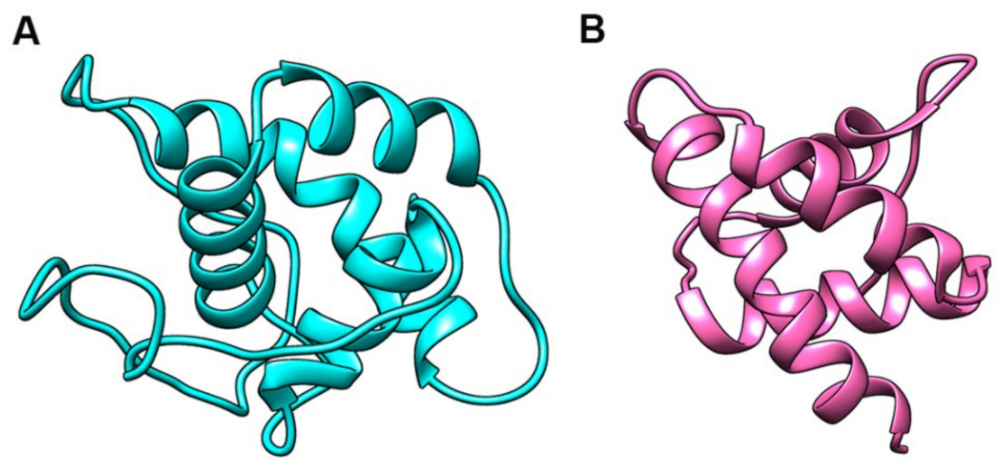

Figure 7. The predicted three-dimensional (3D)-structure of OsHyPRP16 protein. (A) Mature OsHyPRP16 (without signal peptide). (B) Proline-rich region fragment deleted OsHyPRP16.

The docking results for the mature OsHyPRP16 showed strong binding with all the putative ligands, while the fragment deleted OsHyPRP16 was found to exhibit either weak or no binding with the putative ligands (Table 2). These results indicate that the presence of the proline-rich region is essential for the strong binding of mature OsHyPRP16 with the putative ligands. The strong putative binding of mature OsHyPRP16 with fungisterol, ergosterol, N-Acetyl glucosamine (NAG), and N-acetyl muramic acid (NAM) may be involved in the PTI (Table 2), where fungal specific sterols may act as PAMPs to trigger the immune response upon fungal attack. Intriguingly, both the mature as well as fragment deleted OsHyPRP16 were found to show strong binding with JA (Table 2). Previously, the tobacco LTP1, an 8CM containing protein, was found to show binding with JA to form LTP1-JA complex, where the exogenous application of LTP-JA complex confers resistance against Phytophthora parasitica in tobacco plants [73]. In the present study, the predicted binding of OsHyPRP16 with JA and SA, and the induced expression of OsHyPRP16 during SA and methyl-ester of JA (MeJA) treatment (Table 2 and Figure 5E,F) suggests a plausible role of OsHyPRP16 to enhance the resistance against $M$. oryzae in rice through SA- and JA-mediated signaling. 
Table 2. The predicted molecular docking energy level and other features for the interaction of various ligands with mature and fragment deleted OsHyPRP16.

\begin{tabular}{|c|c|c|c|c|c|c|c|}
\hline & Ligand & $\begin{array}{l}\text { Estimated Free } \\
\text { Energy of Binding } \\
\text { (kcal/mol) }\end{array}$ & $\begin{array}{c}\text { vdW + Hbond + } \\
\text { Desolvation } \\
\text { Energy (kcal/mol) }\end{array}$ & $\begin{array}{l}\text { Electrostatic Energy } \\
\text { (kcal/mol) }\end{array}$ & $\begin{array}{c}\text { Total } \\
\text { Intermolecular } \\
\text { Energy (kcal/mol) }\end{array}$ & Frequency (\%) & $\begin{array}{l}\text { Interaction } \\
\text { Surface }\end{array}$ \\
\hline \multicolumn{8}{|c|}{ Mature OsHyPRP16 } \\
\hline \multirow{5}{*}{ Sterols } & Lanosterol & -5.65 & -7.37 & -0.01 & -7.38 & 75 & 869.90 \\
\hline & Desmosterol & -8.88 & -10.61 & -0.04 & -10.65 & 67 & 809.07 \\
\hline & Cholesterol & -9.02 & -10.68 & -0.01 & -10.69 & 57 & 817.93 \\
\hline & Fungisterol & -7.28 & -9.42 & -0.08 & -9.5 & 23 & 819.20 \\
\hline & Ergosterol & -8.08 & -9.52 & -0.01 & -9.53 & 64 & 832.67 \\
\hline \multirow{4}{*}{$\begin{array}{l}\text { Lipids and plant } \\
\text { hormones }\end{array}$} & Linolenic acid & -6.36 & -9.15 & -0.32 & -9.48 & 12 & 677.53 \\
\hline & JA & -6.09 & -6.41 & -0.9 & -7.31 & 90 & 526.62 \\
\hline & MeJA & -5.56 & -6.91 & -0.01 & -6.92 & 33 & 540.28 \\
\hline & SA & -5.54 & -5.66 & -0.11 & -5.77 & 21 & 484.46 \\
\hline \multirow{2}{*}{$\begin{array}{l}\text { Pathogen cell wall } \\
\text { components }\end{array}$} & NAG & -5.5 & -5.64 & -0.11 & -5.75 & 21 & 484.33 \\
\hline & NAM & -6.31 & -6.33 & -0.44 & -6.77 & 48 & 609.37 \\
\hline \multicolumn{8}{|c|}{ Fragment Deleted OsHyPRP16 } \\
\hline \multirow{5}{*}{ Sterols } & Lanosterol & 0.7 & -0.96 & -0.01 & -0.97 & 50 & 867.84 \\
\hline & Desmosterol & 4.86 & 1.84 & -0.05 & -4.89 & 30 & 616.49 \\
\hline & Cholesterol & 8.4 & -3.13 & -0.02 & $-315 \%$ & 40 & 722.06 \\
\hline & Fungisterol & 2.7 & -4.84 & -0.05 & -4.89 & 30 & 716.49 \\
\hline & Ergosterol & 4.08 & 1.26 & 0.06 & 1.32 & 40 & 743.26 \\
\hline \multirow{4}{*}{$\begin{array}{l}\text { Lipids and plant } \\
\text { hormones }\end{array}$} & Linolenic acid & 2.2 & -0.95 & -0.46 & -1.42 & 1 & 600.68 \\
\hline & JA & -5.35 & -6.31 & -0.64 & -6.95 & 70 & 538.91 \\
\hline & MeJA & -2.63 & -4.28 & -0.04 & -4.32 & 24 & 447.63 \\
\hline & SA & -2.3 & -2.78 & -0.13 & -2.91 & 19 & 339.79 \\
\hline \multirow{2}{*}{$\begin{array}{l}\text { Pathogen cell wall } \\
\text { components }\end{array}$} & NAG & -2.38 & -2.72 & -0.05 & -2.76 & 15 & 381.84 \\
\hline & NAM & -2.21 & -3.1 & -0.22 & -3.32 & 4 & 422.58 \\
\hline
\end{tabular}




\section{Materials and Methods}

\subsection{Identification and Sequence Analysis of OsHyPRPs}

The complete set of rice protein sequences were retrieved from the Rice Genome Annotation Project (RGAP) database available at http://rice.plantbiology.msu.edu. All the retrieved rice protein sequences were searched for the HMM profile of Hydrophobic_seed (PF14547), Tryp_alpha_amyl (PF00234), and LTP_2 (PF14368) using “hmmsearch” program of HMMER v 3.1 (http://hmmer.org), with e value $1 \mathrm{e}-4$. The proteins containing any of the HMM profiles were further scanned using PfamScan program (www.ebi.ac.uk/Tools/pfa/pfamscan/), with parameter; -e_seq 1e-04 -e_dom 1e-04 -clan_overlap, to confirm the presence of domains identified through "hmmsearch" program. All the identified putative rice candidate genes were then aligned and manually analyzed for the presence of $8 C M$; $C-X_{n}-C-X_{n}-C C-X_{n}-C X C-X_{n}-C-X_{n}-C$, where $C$ stands for cysteine residue and $X$ stands for any other amino acid residue, while $n$ represents the number of amino acid residues. The proteins lacking the essential 8CM and N-terminal signal peptide (predicted through SignalP-5.0; www.cbs. dtu.dk/services/SignalP/, and TargetP1.1; www.cbs.dtu.dk/services/TargetP/) were identified and then excluded from the further analysis. Furthermore, the proteins containing glycosylphosphatidylinositol (GPI) anchored signals (predicted by big-Pi plant server; mendel.imp.ac.at/gpi/plant_server.html) were also excluded from the analysis. The remaining proteins were then manually inspected for the presence of proline-rich regions, as characterized by presence of tandem repeats of $(X P)_{n}$ and/or $(X P Y)_{n}$ at least for twice [74]. Finally, the shortlisted proteins containing proline-rich regions in between the N-terminal signal peptide and C-terminal 8CM were named as OsHyPRPs. The pI and molecular weight of identified OsHyPRPs was predicted through the Compute pI/Mw tool (web.expasy.org/compute_pi/).

\subsection{Chromosomal Mapping and Gene Duplication Analysis of OsHyPRP Genes}

The genomic coordinates of the OsHyPRP genes were extracted from the general feature format (gff) file (retrieved from https://rapdb.dna.affrc.go.jp/) and were used to schematically represent the intron-exon structure of OsHyPRP genes using Gene Structure Display Server (GSDS; gsds.cbi. pku.edu.cn/). The extracted genomic coordinates were also used to map the location of OsHyPRP genes on all the 12 rice chromosomes using the MapInspect program (www.plantbreeding.wur.nl/uk/ software_mapinspect.html). All the putative genes encoding OsHyPRP proteins were localized on rice chromosomes from $5^{\prime}$ to $3^{\prime}$ based on their genomic coordinates and numbered accordingly from top to bottom on chromosomes 1 to 12 along with prefix OsHyPRP. To analyze the duplication events in OsHyPRP gene family, all-versus-all BLASTP was performed for 66,338 rice protein sequences with parameter: -evalue 1e-10,-outfmt 6,-num_threads 10 and -max_target_seqs 5. The BLASTP results were then used as input for MCScanX [75] to identify the collinear gene pairs of OsHyPRP gene family members in rice with default parameters.

\subsection{Sequence Alignment and Phylogenetic Analysis}

The multiple sequence alignment of full length OsHyPRPs were performed using CLC Genomic Workbench 12.0 (www.qiagenbioinformatics.com/). The 8CM containing C-terminal region of HyPRP proteins of five different plants including rice, Arabidopsis, maize, sorghum, and B. distachyon was extracted and used to find the evolutionary relationship among HyPRP proteins of different plant species. The multiple sequence alignment of deduced sequences was generated using MUSCLE [76], with default parameters, and then used for constructing the phylogenetic tree by employing MEGA software version 6.06 using maximum likelihood method based on the JTT model with 500 bootstrap replicates [77].

\subsection{Biotic and Abiotic Stress and Hormone Treatment}

The rice blast contrasting rice lines, namely, PB1 (blast-susceptible) and PB1+Pi9 (blast-resistant), were used to analyze the expression profile of OsHyPRP genes under different stresses and treatments. 
The seeds of both the rice lines were surface sterilized and germinated on moist filter paper for $4-5$ days at $28^{\circ} \mathrm{C}$. The germinated seeds were transferred to the hydroponic system containing half-strength Murashige and Skoog (MS) medium. For abiotic stress and hormone treatment, two-week-old rice seedlings were treated, separately with salt $(100 \mu \mathrm{M}, \mathrm{NaCl}), \mathrm{ABA}(100 \mu \mathrm{M}), \mathrm{MeJA}(100 \mu \mathrm{M})$, and SA $(100 \mu \mathrm{M})$ by replacing the hydroponic medium with fresh medium containing respective chemicals. Heat and cold stress were given by incubating the two-week-old rice seedlings at $37{ }^{\circ} \mathrm{C}$ and $4{ }^{\circ} \mathrm{C}$, respectively. The leaf samples of treated seedlings were harvested at $0 \mathrm{~h}, 12 \mathrm{~h}, 24 \mathrm{~h}$ and $48 \mathrm{~h}$ intervals after treatment. For biotic stress treatment, we used the method as followed by Jain et al. [43]. Briefly, the 22 days old rice plants were sprayed with M. oryzae (Mo-nwi-53) spore suspension $\left(10^{5} \mathrm{spores} / \mathrm{mL}\right)$ containing $0.25 \%$ gelatin. Leaf tissues of infected rice plants were collected at $12 \mathrm{hpi}, 24 \mathrm{hpi}$, and $48 \mathrm{hpi}$. Some $M$. oryzae infected plants of each rice line were kept under observation and recorded for disease progression after seven days post-inoculation, using $0-5$ disease rating scale [78]. All the treatments were performed in three biological replicates, and the collected tissue samples were immediately frozen in liquid nitrogen and stored at $-80^{\circ} \mathrm{C}$ till further use.

\subsection{RNA Extraction and Real-Time Quantitative Reverse Transcription PCR}

Total RNA from harvested samples were isolated using Sigma's Spectrum Plant Total RNA Kit (Sigma-Aldrich, St. Louis, MO, USA), as per the instruction manual. For first-strand cDNA synthesis, $1 \mu \mathrm{g}$ of total RNA was converted to cDNA using iScript cDNA Synthesis Kit following the manufacturer's instructions (Bio-Rad Laboratories, USA). The primer sequences used for the qRT-PCR analysis of OsHyPRP genes are listed in Supplementary Table S2. The qRT-PCR reactions were performed in $20 \mu \mathrm{L}$ reaction mixture using 2x iQ SYBR Green Supermix (Bio-Rad, California, USA) with universal cycling conditions $\left(95^{\circ} \mathrm{C} ; 5 \mathrm{~min}, 40\right.$ cycles of $95^{\circ} \mathrm{C}$ for $10 \mathrm{~s}$ and $60^{\circ} \mathrm{C}$ for $\left.60 \mathrm{~s}\right)$ and melt curve analysis on a CFX96 Touch Real-Time PCR detection system (Bio-Rad). Each reaction was performed with three technical replicates of each independent biological replicates.

\subsection{Promoter Sequence Analysis of Cis-Regulatory Elements}

The $1.5 \mathrm{~kb}$ region $5^{\prime}$ upstream of the start codon of selected OsHyPRP genes was retrieved from RAP-DB (rapdb.dna.affrc.go.jp/) and analyzed using the PLACE web server (www.hsls.pitt.edu/obrc/ index.php?page=URL1100876009) to find the potential cis-acting regulatory elements.

\subsection{Protein Structure Prediction and Docking Analysis}

The 3D Structures of the candidate protein was predicted through I-Tasser software and further refined by ModRefiner [79,80]. The Ramachandran plot for predicted protein structure was analyzed using Rampage software (mordred.bioc.cam.ac.uk/ \{\}rapper/rampage.php). After validation of protein structure by SAVES server (https://services.mbi.ucla.edu/SAVES/), docking analysis was performed by using molecular modeling simulation software, AutoDock 4, available at Docking Server, as 100 iterations with default parameters [81,82]. For protein-ligand docking analysis, the structure of lipid, sterols and cell wall components was retrieved from PubChem (pubchem.ncbi.nlm.nih.gov) and converted to Docking Server compatible pdb format using babel tool [83].

\section{Conclusions}

In the current study, we systematically identified 45 OsHyPRP genes and characterized them for their chromosomal distribution, gene structure, phylogenetic relationship, duplication analysis, and expression profiling under different experimental conditions. The OsHyPRP genes are mostly intron-less with biased chromosomal distribution and are categorized into seven clades based on their phylogenetic relationship. We also identified some new candidate OsHyPRP genes that were not identified previously. Furthermore, the qRT-PCR based expression profiling revealed the early induced up-regulated expression of OsHyPRP16 under stress conditions and hormone treatment. Additionally, the mature OsHyPRP16 protein was predicted to exhibit a strong binding with various lipids, lipid 
derivatives, fungal cell-wall components, and phytohormones based on the in silico modeling. All these results enable us to put forward the OsHyPRP16 as a promising candidate for future functional analysis that could help to devise strategies for the development of a rice cultivar tolerant to multiple stresses. However, owing to the rapid sequence diversification through ongoing gene duplication in due course of evolution, the functional diversification or possible neofunctionalization of HyPRPs in rice can never be overruled.

Supplementary Materials: The following are available online at http://www.mdpi.com/2223-7747/8/9/343/s1, Figure S1: Collinear genes pairs of OsHyPRP genes on all 12 rice chromosomes. Red lines indicate segmental duplication of OsHyPRP genes on all 12 rice chromosomes and gray lines represent the collinear segments other than of OsHyPRP genes; Figure S2: Tandem and collinear pairs of duplicated OsHyPRP genes in rice genome as predicted through MCScanX; Figure S3: The intron-exon structure of OsHyPRP genes. The genomic coordinates of OsHyPRPs were used to draw the gene structure. The majority of genes were found to be intron-less; Table S1: List of cis-regulatory elements, their sequence, functions and number of elements identified in the $1.5 \mathrm{~kb}$ promoter region of four OsHyPRP genes; Table S2: The sequence of the primers used for qRT-PCR based expression analysis.

Author Contributions: T.R.S. conceived and designed the project; R.K., P.A., and G.K. performed in silico analysis of HyPRPs gene family; R.K. and R.J. performed the structure prediction and docking analysis; P.J. analyzed the RNA-Seq expression data; R.K. performed all major experiments; R.K. and G.K. analyzed the data; T.R.S., K.S., R.K., and G.K. wrote the manuscript. All authors have read and approved the manuscript.

Funding: T.R.S. is thankful to the Department of Science and Technology, Government of India for the J.C. Bose National Fellowship and financial support for this work. R.K. is thankful to the Indian Council of Medical Research, India for providing fellowship.

Acknowledgments: The authors would like to thank Adam Lazere, National Eye Institute-National Institute of Health, Bethesda, Maryland, USA, for improving the article's readability and English language.

Conflicts of Interest: The authors have declared that they have no conflicts of interest.

\section{References}

1. Jones, J.D.G.; Dangl, J.L. The plant immune system. Nature 2006, 444, 323. [CrossRef] [PubMed]

2. Singh, P.; Nag, A.; Arya, P.; Kapoor, R.; Singh, A.; Jaswal, R.; Sharma, T. Prospects of understanding the molecular biology of disease resistance in rice. Int. J. Mol. Sci. 2018, 19, 1141. [CrossRef] [PubMed]

3. Ali, S.; Ganai, B.A.; Kamili, A.N.; Bhat, A.A.; Mir, Z.A.; Bhat, J.A.; Tyagi, A.; Islam, S.T.; Mushtaq, M.; Yadav, P. Pathogenesis-related proteins and peptides as promising tools for engineering plants with multiple stress tolerance. Microbiol. Res. 2018, 212, 29-37. [CrossRef] [PubMed]

4. Diaz, I. Plant Defense Genes against Biotic Stresses. Int. J. Mol. Sci. 2018, 19, 2446. [CrossRef] [PubMed]

5. Van Loon, L.C.; Rep, M.; Pieterse, C.M.J. Significance of inducible defense-related proteins in infected plants. Annu. Rev. Phytopathol. 2006, 44, 135-162. [CrossRef] [PubMed]

6. Shewry, P.R.; Beaudoin, F.; Jenkins, J.; Griffiths-Jones, S.; Mills, E.N.C. Plant protein families and their relationships to food allergy. Biochem. Soc. Trans. 2002, 30, 906-910. [CrossRef] [PubMed]

7. Radauer, C.; Breiteneder, H. Evolutionary biology of plant food allergens. J. Allergy Clin. Immunol. 2007, 120, 518-525. [CrossRef] [PubMed]

8. José-Estanyol, M.; Gomis-Rüth, F.X.; Puigdomènech, P. The eight-cysteine motif, a versatile structure in plant proteins. Plant Physiol. Biochem. 2004, 42, 355-365. [CrossRef] [PubMed]

9. Dvořáková, L.; Srba, M.; Opatrny, Z.; Fischer, L. Hybrid proline-rich proteins: Novel players in plant cell elongation? Ann. Bot. 2011, 109, 453-462. [CrossRef] [PubMed]

10. Josè, M.; Puigdomènech, P. Structure and expression of genes coding for structural proteins of the plant cell wall. New Phytol. 1993, 125, 259-282. [CrossRef]

11. Josè-Estanyol, M.; Puigdomènech, P. Plant cell wall glycoproteins and their genes. Plant Physiol. Biochem. 2000, 38, 97-108. [CrossRef]

12. Dvořáková, L.; Cvrčková, F.; Fischer, L. Analysis of the hybrid proline-rich protein families from seven plant species suggests rapid diversification of their sequences and expression patterns. BMC Genom. 2007, 8, 412. [CrossRef] [PubMed]

13. Yeats, T.H.; Rose, J.K.C. The biochemistry and biology of extracellular plant lipid-transfer proteins (LTPs). Protein Sci. 2008, 17, 191-198. [CrossRef] [PubMed] 
14. Liu, F.; Zhang, X.; Lu, C.; Zeng, X.; Li, Y.; Fu, D.; Wu, G. Non-specific lipid transfer proteins in plants: Presenting new advances and an integrated functional analysis. J. Exp. Bot. 2015, 66, 5663-5681. [CrossRef] [PubMed]

15. Finkina, E.I.; Melnikova, D.N.; Bogdanov, I.V. Lipid transfer proteins as components of the plant innate immune system: Structure, functions, and applications. Acta Naturae 2016, 8, 29. [CrossRef]

16. Castonguay, Y.; Laberge, S.; Nadeau, P.; Vézina, L.-P. A cold-induced gene from Medicago sativa encodes a bimodular protein similar to developmentally regulated proteins. Plant Mol. Biol. 1994, 24, 799-804. [CrossRef]

17. Deutch, C.E.; Winicov, I. Post-transcriptional regulation of a salt-inducible alfalfa gene encoding a putative chimeric proline-rich cell wall protein. Plant Mol. Biol. 1995, 27, 411-418. [CrossRef]

18. Goodwin, W.; Pallas, J.A.; Jenkins, G.I. Transcripts of a gene encoding a putative cell wall-plasma membrane linker protein are specifically cold-induced in Brassica napus. Plant Mol. Biol. 1996, 31, 771-781. [CrossRef]

19. Zhang, Y.; Schläppi, M. Cold responsive EARLI1 type HyPRPs improve freezing survival of yeast cells and form higher order complexes in plants. Planta 2007, 227, 233-243. [CrossRef]

20. Priyanka, B.; Sekhar, K.; Reddy, V.D.; Rao, K.V. Expression of pigeonpea hybrid-proline-rich protein encoding gene (CcHyPRP) in yeast and Arabidopsis affords multiple abiotic stress tolerance. Plant Biotechnol. J. 2010, 8, 76-87. [CrossRef]

21. Huang, G.; Gong, S.; Xu, W.; Li, P.; Zhang, D.; Qin, L.; Li, W.; Li, X. GhHyPRP4, a cotton gene encoding putative hybrid proline-rich protein, is preferentially expressed in leaves and involved in plant response to cold stress. Acta Biochim. Biophys. Sin. 2011, 43, 519-527. [CrossRef] [PubMed]

22. Qin, L.-X.; Zhang, D.-J.; Huang, G.-Q.; Li, L.; Li, J.; Gong, S.-Y.; Li, X.-B.; Xu, W.-L. Cotton GhHyPRP3 encoding a hybrid proline-rich protein is stress inducible and its overexpression in Arabidopsis enhances germination under cold temperature and high salinity stress conditions. Acta Physiol. Plant. 2013, 35, 1531-1542. [CrossRef]

23. Tan, J.; Zhuo, C.; Guo, Z. Nitric oxide mediates cold-and dehydration-induced expression of a novel MfHyPRP that confers tolerance to abiotic stress. Physiol. Plant. 2013, 149, 310-320. [PubMed]

24. Liu, D.Q.; Han, Q.; Shah, T.; Chen, C.Y.; Wang, Q.; Tang, B.F.; Ge, F. A hybrid proline-rich cell-wall protein gene JsPRP1 from Juglans sigillata Dode confers both biotic and abiotic stresses in transgenic tobacco plants. Trees 2018, 32, 1199-1209. [CrossRef]

25. Jose-Estanyol, M.; Ruiz-Avila, L.; Puigdomènech, P. A maize embryo-specific gene encodes a proline-rich and hydrophobic protein. Plant Cell 1992, 4, 413-423. [CrossRef] [PubMed]

26. He, C.-Y.; Zhang, J.-S.; Chen, S.-Y. A soybean gene encoding a proline-rich protein is regulated by salicylic acid, an endogenous circadian rhythm and by various stresses. Theor. Appl. Genet. 2002, 104, 1125-1131. [CrossRef] [PubMed]

27. Bücker Neto, L.; de Oliveira, R.R.; Wiebke-Strohm, B.; Bencke, M.; Weber, R.L.M.; Cabreira, C.; Abdelnoor, R.V.; Marcelino, F.C.; Zanettini, M.H.B.; Passaglia, L.M.P. Identification of the soybean HyPRP family and specific gene response to Asian soybean rust disease. Genet. Mol. Biol. 2013, 36, 214-224. [CrossRef]

28. Li, B.-C.; Zhang, C.; Chai, Q.-X.; Han, Y.-Y.; Wang, X.-Y.; Liu, M.-X.; Feng, H.; Xu, Z.-Q. Plasmalemma localisation of DOUBLE HYBRID PROLINE-RICH PROTEIN 1 and its function in systemic acquired resistance of Arabidopsis thaliana. Funct. Plant Biol. 2014, 41, 768-779. [CrossRef]

29. Li, J.; Ouyang, B.; Wang, T.; Luo, Z.; Yang, C.; Li, H.; Sima, W.; Zhang, J.; Ye, Z. HyPRP1 gene suppressed by multiple stresses plays a negative role in abiotic stress tolerance in tomato. Front. Plant Sci. 2016, 7, 967. [CrossRef]

30. Wu, H.; Zou, J.; May, B.; Gu, Q.; Cheung, A.Y. A tobacco gene family for flower cell wall proteins with a proline-rich domain and a cysteine-rich domain. Proc. Natl. Acad. Sci. USA 1993, 90, 6829-6833. [CrossRef]

31. Holk, A.; Klumpp, L.; Scherer, G.F.E. A cell wall protein down-regulated by auxin suppresses cell expansion in Daucus carota (L.). Plant Mol. Biol. 2002, 50, 295-305. [CrossRef] [PubMed]

32. Blanco-Portales, R.; Lopez-Raez, J.A.; Bellido, M.L.; Moyano, E.; Dorado, G.; Gonzalez-Reyes, J.A.; Caballero, J.L.; Munoz-Blanco, J. A strawberry fruit-specific and ripening-related gene codes for a HyPRP protein involved in polyphenol anchoring. Plant Mol. Biol. 2004, 55, 763-780. [CrossRef] [PubMed]

33. Bouton, S.; Viau, L.; Lelièvre, E.; Limami, A.M. A gene encoding a protein with a proline-rich domain (MtPPRD1), revealed by suppressive subtractive hybridization (SSH), is specifically expressed in the Medicago truncatula embryo axis during germination. J. Exp. Bot. 2005, 56, 825-832. [CrossRef] [PubMed] 
34. Sundaresan, S.; Philosoph-Hadas, S.; Ma, C.; Jiang, C.-Z.; Riov, J.; Mugasimangalam, R.; Kochanek, B.; Salim, S.; Reid, M.S.; Meir, S. The Tomato Hybrid Proline-rich Protein regulates the abscission zone competence to respond to ethylene signals. Hortic. Res. 2018, 5, 28. [CrossRef] [PubMed]

35. Bubier, J.; Schläppi, M. Cold induction of EARLI1, a putative Arabidopsis lipid transfer protein, is light and calcium dependent. Plant. Cell Environ. 2004, 27,929-936. [CrossRef]

36. Peng, T.; Jia, M.-M.; Liu, J.-H. RNAi-based functional elucidation of PtrPRP, a gene encoding a hybrid proline rich protein, in cold tolerance of Poncirus trifoliata. Front. Plant Sci. 2015, 6, 808. [CrossRef] [PubMed]

37. Mellacheruvu, S.; Tamirisa, S.; Vudem, D.R.; Khareedu, V.R. Pigeonpea hybrid-proline-rich protein (CcHyPRP) confers biotic and abiotic stress tolerance in transgenic rice. Front. Plant Sci. 2016, 6, 1167. [CrossRef]

38. Jung, H.W.; Tschaplinski, T.J.; Wang, L.; Glazebrook, J.; Greenberg, J.T. Priming in systemic plant immunity. Science 2009, 324, 89-91. [CrossRef]

39. Yeom, S.; Seo, E.; Oh, S.; Kim, K.W.; Choi, D. A common plant cell-wall protein HyPRP1 has dual roles as a positive regulator of cell death and a negative regulator of basal defense against pathogens. Plant J. 2012, 69, 755-768. [CrossRef]

40. Yang, J.; Zhang, Y.; Wang, X.; Wang, W.; Li, Z.; Wu, J.; Wang, G.; Wu, L.; Zhang, G.; Ma, Z. HyPRP1 performs a role in negatively regulating cotton resistance to $V$. dahliae via the thickening of cell walls and ROS accumulation. BMC Plant Biol. 2018, 18, 339. [CrossRef]

41. Imam, J.; Alam, S.; Mandal, N.P.; Variar, M.; Shukla, P. Molecular screening for identification of blast resistance genes in North East and Eastern Indian rice germplasm (Oryza sativa L.) with PCR based makers. Euphytica 2014, 196, 199-211. [CrossRef]

42. Khanna, A.; Sharma, V.; Ellur, R.K.; Shikari, A.B.; Krishnan, S.G.; Singh, U.D.; Prakash, G.; Sharma, T.R.; Rathour, R.; Variar, M. Development and evaluation of near-isogenic lines for major blast resistance gene (s) in Basmati rice. Theor. Appl. Genet. 2015, 128, 1243-1259. [CrossRef] [PubMed]

43. Jain, P.; Singh, P.K.; Kapoor, R.; Khanna, A.; Solanke, A.U.; Krishnan, S.G.; Singh, A.K.; Sharma, V.; Sharma, T.R. Understanding host-pathogen interactions with expression profiling of NILs carrying rice-blast resistance Pi9 gene. Front. Plant Sci. 2017, 8, 93. [CrossRef] [PubMed]

44. Boutrot, F.; Chantret, N.; Gautier, M.-F. Genome-wide analysis of the rice and Arabidopsis non-specific lipid transfer protein (nsLtp) gene families and identification of wheat nsLtp genes by EST data mining. BMC Genomics 2008, 9, 86. [CrossRef] [PubMed]

45. Yu, J.; Wang, J.; Lin, W.; Li, S.; Li, H.; Zhou, J.; Ni, P.; Dong, W.; Hu, S.; Zeng, C. The genomes of Oryza sativa: A history of duplications. PLoS Biol. 2005, 3, e38. [CrossRef] [PubMed]

46. Seherm, H.; Coakley, S.M. Plant pathogens in a changing world. Australas. Plant Pathol. 2003, 32, 157-165. [CrossRef]

47. Pandey, P.; Irulappan, V.; Bagavathiannan, M.V.; Senthil-Kumar, M. Impact of combined abiotic and biotic stresses on plant growth and avenues for crop improvement by exploiting physio-morphological traits. Front. Plant Sci. 2017, 8, 537. [CrossRef] [PubMed]

48. Creissen, H.E.; Jorgensen, T.H.; Brown, J.K.M. Impact of disease on diversity and productivity of plant populations. Funct. Ecol. 2016, 30, 649-657. [CrossRef]

49. Fernandez, J.; Orth, K. Rise of a cereal killer: The biology of Magnaporthe oryzae biotrophic growth. Trends Microbiol. 2018, 26, 582-597. [CrossRef] [PubMed]

50. Li, L.; Zhang, C.; Xu, D.; Schläppi, M.; Xu, Z.-Q. Expression of recombinant EARLI1, a hybrid proline-rich protein of Arabidopsis, in Escherichia coli and its inhibition effect to the growth of fungal pathogens and Saccharomyces cerevisiae. Gene 2012, 506, 50-61. [CrossRef]

51. Guy, C.; Kaplan, F.; Kopka, J.; Selbig, J.; Hincha, D.K. Metabolomics of temperature stress. Physiol. Plant. 2008, 132, 220-235. [CrossRef] [PubMed]

52. Aghamolki, M.T.K.; Yusop, M.K.; Oad, F.C.; Zakikhani, H.; Jaafar, H.Z.; Kharidah, S.; Musa, M.H. Heat stress effects on yield parameters of selected rice cultivars at reproductive growth stages. J. Food Agric. Env. 2014, 12, 741-746.

53. Hatfield, J.L.; Prueger, J.H. Temperature extremes: Effect on plant growth and development. Weather Clim. Extrem. 2015, 10, 4-10. [CrossRef]

54. Rodríguez, V.M.; Soengas, P.; Alonso-Villaverde, V.; Sotelo, T.; Cartea, M.E.; Velasco, P. Effect of temperature stress on the early vegetative development of Brassica oleracea L. BMC Plant Biol. 2015, 15, 145. [CrossRef] [PubMed] 
55. Jing, P.; Wang, D.; Zhu, C.; Chen, J. Plant physiological, morphological and yield-related responses to night temperature changes across different species and plant functional types. Front. Plant Sci. 2016, 7, 1774. [CrossRef] [PubMed]

56. Nievola, C.C.; Carvalho, C.P.; Carvalho, V.; Rodrigues, E. Rapid responses of plants to temperature changes. Temperature 2017, 4, 371-405. [CrossRef] [PubMed]

57. Srinath, T.; Reddy, V.D.; Rao, K.V. Isolation and functional characterization of a novel stress inducible promoter from pigeonpea (Cajanus cajan L.). Plant Cell Tissue Organ Cult. 2017, 128, 457-468. [CrossRef]

58. Wilkosz, R.; Schläppi, M. A gene expression screen identifies EARLI1 as a novel vernalization-responsive gene in Arabidopsis thaliana. Plant Mol. Biol. 2000, 44, 777-787. [CrossRef]

59. Xu, D.; Huang, X.; Xu, Z.-Q.; Schläppi, M. The HyPRP gene EARLI1 has an auxiliary role for germinability and early seedling development under low temperature and salt stress conditions in Arabidopsis thaliana. Planta 2011, 234, 565-577. [CrossRef]

60. Xu, Z.-Y.; Zhang, X.; Schläppi, M.; Xu, Z.-Q. Cold-inducible expression of AZI1 and its function in improvement of freezing tolerance of Arabidopsis thaliana and Saccharomyces cerevisiae. J. Plant Physiol. 2011, 168, 1576-1587. [CrossRef]

61. Pitzschke, A.; Datta, S.; Persak, H. Salt stress in Arabidopsis: Lipid transfer protein AZI1 and its control by mitogen-activated protein kinase MPK3. Mol. Plant 2014, 7, 722-738. [CrossRef] [PubMed]

62. Vickers, C.E.; Gershenzon, J.; Lerdau, M.T.; Loreto, F. A unified mechanism of action for volatile isoprenoids in plant abiotic stress. Nat. Chem. Biol. 2009, 5, 283-291. [CrossRef] [PubMed]

63. Gray, W.M. Hormonal regulation of plant growth and development. PLoS Biol. 2004, 2, e311. [CrossRef] [PubMed]

64. Kamiya, Y. Plant hormones: Versatile regulators of plant growth and development. Annu. Rev. Plant Biol. 2010, 60. [CrossRef]

65. Wang, Y.H.; Irving, H.R. Developing a model of plant hormone interactions. Plant Signal. Behav. 2011, 6, 494-500. [CrossRef] [PubMed]

66. Munné-Bosch, S.; Müller, M. Hormonal cross-talk in plant development and stress responses. Front. Plant Sci. 2013, 4, 529. [CrossRef] [PubMed]

67. Nejat, N.; Mantri, N. Plant immune system: Crosstalk between responses to biotic and abiotic stresses the missing link in understanding plant defence. Curr. Issues Mol. Biol. 2017, 23, 1-16. [CrossRef] [PubMed]

68. Ku, Y.-S.; Sintaha, M.; Cheung, M.-Y.; Lam, H.-M. Plant hormone signaling crosstalks between biotic and abiotic stress responses. Int. J. Mol. Sci. 2018, 19, 3206. [CrossRef] [PubMed]

69. Kunkel, B.N.; Brooks, D.M. Cross talk between signaling pathways in pathogen defense. Curr. Opin. Plant Biol. 2002, 5, 325-331. [CrossRef]

70. Kohli, A.; Sreenivasulu, N.; Lakshmanan, P.; Kumar, P.P. The phytohormone crosstalk paradigm takes center stage in understanding how plants respond to abiotic stresses. Plant Cell Rep. 2013, 32, 945-957. [CrossRef]

71. Pandey, G.K.; Grant, J.J.; Cheong, Y.H.; Kim, B.G.; Li, L.; Luan, S. ABR1, an APETALA2-domain transcription factor that functions as a repressor of ABA response in Arabidopsis. Plant Physiol. 2005, 139, 1185-1193. [CrossRef] [PubMed]

72. Hernandez-Garcia, C.M.; Finer, J.J. Identification and validation of promoters and cis-acting regulatory elements. Plant Sci. 2014, 217, 109-119. [CrossRef] [PubMed]

73. Buhot, N.; Gomes, E.; Milat, M.-L.; Ponchet, M.; Marion, D.; Lequeu, J.; Delrot, S.; Coutos-Thévenot, P.; Blein, J.-P. Modulation of the biological activity of a tobacco LTP1 by lipid complexation. Mol. Biol. Cell 2004, 15, 5047-5052. [CrossRef] [PubMed]

74. Williamson, M.P. The structure and function of proline-rich regions in proteins. Biochem. J. 1994, $297,249$. [CrossRef] [PubMed]

75. Wang, Y.; Tang, H.; DeBarry, J.D.; Tan, X.; Li, J.; Wang, X.; Lee, T.; Jin, H.; Marler, B.; Guo, H. MCScanX: A toolkit for detection and evolutionary analysis of gene synteny and collinearity. Nucleic Acids Res. 2012, 40, e49. [CrossRef]

76. Edgar, R.C. MUSCLE: Multiple sequence alignment with high accuracy and high throughput. Nucleic Acids Res. 2004, 32, 1792-1797. [CrossRef] [PubMed]

77. Tamura, K.; Stecher, G.; Peterson, D.; Filipski, A.; Kumar, S. MEGA6: Molecular evolutionary genetics analysis version 6.0. Mol. Biol. Evol. 2013, 30, 2725-2729. [CrossRef] 
78. Bonman, J.M.; De Dios, T.I.V.; Khin, M.M. Physiologic specialization of Pyricularia oryzae in the Philippines. Plant Dis. 1986, 70, 767-769. [CrossRef]

79. Yang, J.; Yan, R.; Roy, A.; Xu, D.; Poisson, J.; Zhang, Y. The I-TASSER Suite: Protein structure and function prediction. Nat. Methods 2015, 12,7-8. [CrossRef]

80. Xu, D.; Zhang, J.; Roy, A.; Zhang, Y. Automated protein structure modeling in CASP9 by I-TASSER pipeline combined with QUARK-based ab initio folding and FG-MD-based structure refinement. Proteins Struct. Funct. Bioinform. 2011, 79, 147-160. [CrossRef]

81. Huey, R.; Morris, G.M.; Olson, A.J.; Goodsell, D.S. A semiempirical free energy force field with charge-based desolvation. J. Comput. Chem. 2007, 28, 1145-1152. [CrossRef] [PubMed]

82. Bikadi, Z.; Hazai, E. Application of the PM6 semi-empirical method to modeling proteins enhances docking accuracy of AutoDock. J. Cheminform. 2009, 1, 15. [CrossRef] [PubMed]

83. O’Boyle, N.M.; Banck, M.; James, C.A.; Morley, C.; Vandermeersch, T.; Hutchison, G.R. Open Babel: An open chemical toolbox. J. Cheminform. 2011, 3, 33. [CrossRef] [PubMed]

(C) 2019 by the authors. Licensee MDPI, Basel, Switzerland. This article is an open access article distributed under the terms and conditions of the Creative Commons Attribution (CC BY) license (http://creativecommons.org/licenses/by/4.0/). 